Karl-Franzens Universität Graz

Technische Universität Graz

Medizinische Universität Graz

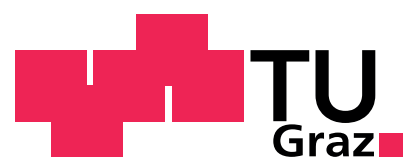

\title{
A bilevel optimization approach for parameter learning in variational models
}
K. Kunisch
T. Pock 
SFB sponsors:

- Austrian Science Fund (FWF)

- University of Graz

- Graz University of Technology

- Medical University of Graz

- Government of Styria

- City of Graz

Das Land

Steiermark

Stadt $\mathbf{G} \mathbf{R}$ A Z $\mathbf{Z}$ Wissenschaft 


\title{
A bilevel optimization approach for parameter learning in variational models
}

\author{
Karl Kunisch* Thomas Pock ${ }^{\dagger}$
}

July 9, 2012

\begin{abstract}
In this work we consider the problem of parameter learning for variational image denoising models. The learning problem is formulated as a bilevel optimization problem, where the lower level problem is given by the variational model and the higher level problem is expressed by means of a loss function that penalizes errors between the solution of the lower level problem and the ground truth data. We consider a class of image denoising models incorporating $\ell_{p}$-norm based analysis priors using a fixed set of linear operators. We devise semi-smooth Newton methods to solve the resulting non-smooth bilevel optimization problems and show that the optimized image denoising models can achieve state-of-the-art performance.
\end{abstract}

Keywords: Regularization parameter, image denoising, learning theory, non-differentiable optimization, bilevel optimization, semi-smooth Newton algorithm.

AMS Subject Classification: 49J52, 49N45, 68U10.

\section{Introduction}

Variational approaches had great success in solving inverse problems in imaging, such as image restoration, optical flow and stereo vision. The fundamen-

\footnotetext{
* Institute of Mathematics and Scientific Computing, University of Graz, Heinrichstraße 36, Graz, A-8010, Austria. Research in part supported by the Austrian Science Fund (FWF) under grant SFB F32 (SFB "Mathematical Optimization and Applications in Biomedical Sciences")

e-mail: karl.kunisch@uni-graz.at

${ }^{\dagger}$ Institute for Computer Graphics and Vision, Graz University of Technology, Inffeldgasse 16, A-8010 Graz, Austria.

e-mail: pock@icg.tugraz.at
} 
tal principle behind these approaches is to devise the solution of the inverse problem as the minimizer of an energy functional, which is designed such that its minimum-energy state reflects the characteristic properties of the solution. For example, popular priors assume that the solution is piecewise constant or piecewise smooth.

Usually, variational models incorporate a number of free parameters. These parameters are used for example to tradeoff between regularization and data fidelity or to locally adapt the variational model to the input data. Selecting optimal parameters is by far not trivial. A possible procedure to determine these free parameters is to evaluate the performance of the variational model on some test data with known optimal solution by performing an exhaustive search over a range of useful parameters settings. This is tedious and becomes infeasible already for more than two or three parameters.

In this work a systematic approach for the above procedure will be provided. We cast parameter selection as a learning problem. Given a certain variational model, the task consists in learning the parameters such that the variational model minimizes a certain loss functional on a training database. This naturally leads to a bi-level optimization problem of the following form:

$$
\left\{\begin{array}{l}
\min _{\vartheta \geq 0} \mathcal{E}(x(\vartheta)) \\
\text { subject to } x(\vartheta) \in \arg \min _{x} \mathcal{F}(x, \vartheta)
\end{array}\right.
$$

The bi-level problem consists in a lower-level optimization whose solution $x(\vartheta)$ is an argument of the higher-level minimization problem. The aim of the bi-level problem is then to find a parameter vector $\vartheta$ such that $\mathcal{E}(x(\vartheta))$ attains a minimum value.

Concerning the choice of regularization parameters the literature typically distinguishes between a-posteriori and a-priori parameter rules, as well as error-free parameter choice rules, see e.g. [10, 12], and the references cited there. The discrepancy principle is a prominent example for an a-posteriori rule, where the regularization parameter is determined such that the data fidelity term at the optimum equals the size of the noise level. Here we require knowledge of the noise level as well as the noisy data. A-priori rules determine the regularization parameter solely from knowledge of the noise level. The class of parameter free methods includes generalized cross validation and balancing principles between the error in the fidelity and the regularization terms. Most of the work on parameter choice techniques addresses the case of a single scalar parameter.

Bilevel optimization problems are an active research area in their own right, see e.g. [2] and the references provided there. Here we only analyze the 
specific bilevel problem (1.1) to the extent that is required to propose and investigate numerical methods for its solution. In this work the functional $\mathcal{E}$ of the upper level problem will be smooth while for the lower level problem we distinguish between a smooth quadratic and the non-smooth $\ell_{1}$ and $\ell_{\frac{1}{2}}$ cases.

For the application of image restoration, bilevel optimization has been used by Tappen et al. in $[28,29,27]$ to learn the parameters of different Markov random field models. In particular, they showed that bilevel optimization provides an effective learning method, as it overcomes the typical problems of classical probabilistic learning methods that require to compute the partition function of the underyling probability density function. However, while Tappen et al. used gradient methods for learning that do not come along with any convergence guarantees, we propose fast Newton methods that come along with locally super-linear convergence. It will turn out that our proposed Newton algorithms do not only provide an effective learning framework but also lead to image restoration results superior to that reported in [27]. We mainly attribute this fact to the ability of our proposed algorithms to be more successful in finding a (local) minimizer of the bilevel optimization problems, than the gradient methods used in [27]. In [24], a bilevel learning approach was proposed for sparse analysis prior learning using an $\ell_{1}$ model. The approach is similar to [27] as it uses implicit differentiation to compute the gradient of the higher level problem with respect to the learning parameters.

Let us give a brief summary of the contents of the following sections. In Section 2 we present the precise problem statement and provide some preliminaries. The smooth case with a single as well as multiple priors is aqnalyzed in Section 3. We investigate aspects of the geometry of the value functional $\mathcal{E}$ and develop a Newton algorithm for the solution of the inequality constrained problem (1.1). Section 4 is devoted to existence of (1.1) and the derivation of an optimality condition by means of a regularisation procedure for the case when the lower level problem is non-smooth. The regularized problems are semi-smooth and thus we propose a semi-smooth Newton algorithm for their solution. Numerical experiments for a wide variety of priors and for images of different qualitative features are presented in Section 5. 


\section{Preliminaries}

In this work we put our emphasis on the following class of problems:

$$
\left\{\begin{array}{l}
\min _{\vartheta \geq 0} \mathcal{E}(x(\vartheta))=\|x(\vartheta)-g\|_{2}^{2} \\
\text { subject to } x(\vartheta)=\arg \min _{x} \mathcal{F}(x, \vartheta)=\frac{1}{p} \sum_{k=1}^{q} \vartheta_{k}\left\|K_{k} x\right\|_{p}^{p}+\frac{1}{2}\|x-f\|_{2}^{2} .
\end{array}\right.
$$

The lower-level optimization problems $\mathcal{F}(x, \vartheta)$ consists of a data and of a regularization term. The data term penalizes the squared $\ell_{2}$-norm of the discrepancy between the noisy image $f \in \mathbb{R}^{n}$ and the unknown image $x \in \mathbb{R}^{n}$. The regularization term is a sum of $q \geq 1$ so-called analysis based priors (see e.g. [31]), penalizing the $\ell_{p}^{p}$-norms

$$
\left\|K_{k} x\right\|_{p}^{p}=\sum_{i=1}^{n}\left|\left(K_{k} x\right)_{i}\right|^{p}
$$

of the result of applying linear operators $K_{k} \in \mathbb{R}^{m \times n}, 1 \leq k \leq q$ to $x$. We shall consider primarily the cases $p \in\{1,2\}$, and in numerical experiments also $p=\frac{1}{2}$. The importance of the priors $\left\|K_{k} x\right\|_{p}^{p}, 1 \leq k \leq q$ are weighted by parameters $\vartheta_{k} \geq 0$, which are assembled in a parameter vector $\vartheta=$ $\left(\vartheta_{1}, \ldots, \vartheta_{q}\right)$.

The higher-level optimization problem $\mathcal{E}(x(\vartheta))$ penalizes the discrepancy between the minimizer of the lower level optimization problem $x(\vartheta)$ and given ground truth data $g \in \mathbb{R}^{n}$ by means of the squared 2-norm. In some situations we will eliminate $x$ which leads to a reduced single-level optimization problem $\mathcal{E}(\vartheta)$, as opposed to the bi-level optimization problem $\mathcal{E}(x(\vartheta))$.

We frequently make use of a standard inner product on $\mathbb{R}^{n}$ denoted by $\langle\cdot, \cdot\rangle$, which induces the 2-norm $\|\cdot\|_{2}=\langle\cdot, \cdot\rangle^{\frac{1}{2}}$. We further denote by $\operatorname{ker}(K)=\left\{x \in \mathbb{R}^{n}: K x=0\right\}$ the kernel of $K$ and by $\operatorname{ran}(K)=\{K x: x \in$ $\left.\mathbb{R}^{n}\right\}$ the range or column space of $K$. The operation max on a vector $x \in \mathbb{R}^{n}$ is understood to operate elementwise, i.e.

$$
\max (0, x)=\left(\max \left(0, x_{1}\right), \ldots, \max \left(0, x_{n}\right)\right) .
$$

To obtain some insight into the cost functional $\mathcal{E}$ associated to (1.1) let us investigate the scalar-valued case, i.e. $x, f, g \in \mathbb{R}, q=1$ and $K_{1}=1$. For $p=2$ and by combining the lower level problem with the higher level problem we arrive at the single level problem

$$
\min _{\vartheta \geq 0} \mathcal{E}_{\ell_{2}}(\vartheta)=\left(\frac{f}{1+\vartheta}-g\right)^{2}
$$




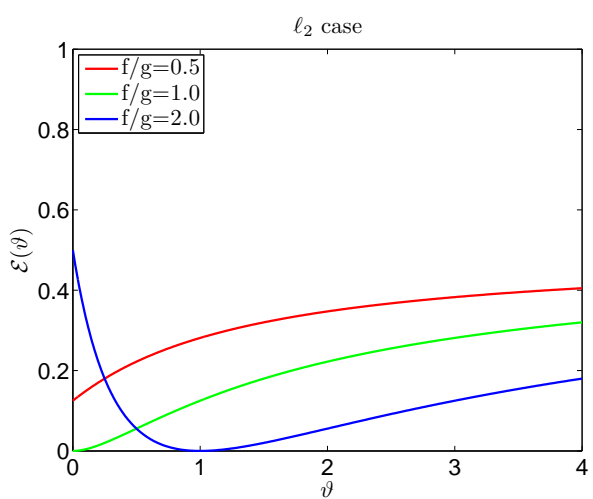

(a)

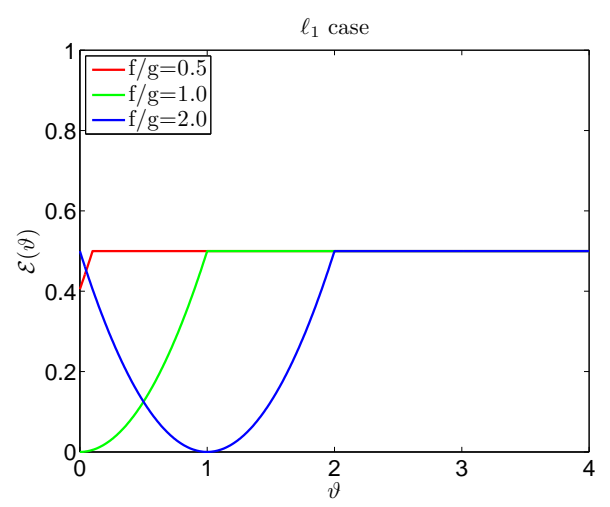

(b)

Figure 1: Shape of the reduced single level problems for the $\ell_{2}$ and the $\ell_{1}$ case.

We plot its graph for the scalar-valued case in Figure 1 (a) for various choices of the ratio $f / g$. It is easy to show that all sublevel sets of $\mathcal{E}_{\ell_{2}}(\vartheta)$ are convex and hence $\mathcal{E}_{\ell_{2}}(\vartheta)$ is quasiconvex. In the case of $p=1$ the single level problem becomes

$$
\min _{\vartheta \geq 0} \mathcal{E}_{\ell_{1}}(\vartheta)=(\max (0,|f|-\vartheta) \operatorname{sgn}(f)-g)^{2},
$$

which is non-smooth since the solution to the lower level problem coincides with $f$ for all $\vartheta$ larger than a threshold value. Figure 1 (b) shows $\mathcal{E}_{\ell_{1}}(\vartheta)$ again for various choices of the ratio $f / g$. Again, it can be shown that $\mathcal{E}_{\ell_{1}}(\vartheta)$ is quasiconvex. The quasi-convexity is of interest since it improves the chance that optimization algorithms find the optimal regularization parameters of the models. In the following section a sufficient condition is found that the guarantees this property also for the multi-dimensional, single prior case for $\ell^{2}$ models.

\section{The $\ell_{2}$ model}

\subsection{Single prior}

Let us first consider the most simple instance of (2.1) where we set $p=2$ and $q=1$, which corresponds to computing the optimal regularization parameter 
in a classical Tikhonov regularization functional:

$$
\left\{\begin{array}{l}
\min _{\vartheta \geq 0} \mathcal{E}(x(\vartheta))=\|x(\vartheta)-g\|_{2}^{2} \\
\text { subject to } x(\vartheta)=\arg \min _{x} \frac{\vartheta}{2}\|K x\|_{2}^{2}+\frac{1}{2}\|x-f\|_{2}^{2},
\end{array}\right.
$$

Solving the lower-level optimization problem we find $x(\vartheta)=\left(I+\vartheta K^{T} K\right)^{-1} f$ and hence (3.1) is equivalent to

$$
\min _{\vartheta \geq 0} \mathcal{E}(\vartheta)=\left\|\left(I+\vartheta K^{T} K\right)^{-1} f-g\right\|_{2}^{2}
$$

It will be convenient to introduce $\mathcal{K}=K^{T} K \in \mathbb{R}^{n \times n}$. Every element $x \in \mathbb{R}^{n}$ can be uniquely decomposed as

$$
x=x^{N}+x^{\perp} \in \operatorname{ker}(\mathcal{K}) \oplus \operatorname{ran}(\mathcal{K}) .
$$

In our first result, we give a condition which ensures the existence of a minimizer of (3.2).

Proposition 3.1. If $\left\|f^{\perp}-g^{\perp}\right\|_{2}<\left\|g^{\perp}\right\|_{2}$, then (3.2) admits a solution $\vartheta^{*} \geq 0$. If moreover $\langle\mathcal{K} f, f-g\rangle>0$, then $\vartheta^{*}>0$.

Proof. Let $\left\{\vartheta_{n}\right\}_{n=1}^{\infty}$, with $\vartheta_{n} \geq 0$ be a minimizing sequence, i.e.

$$
\lim _{n \rightarrow \infty} \mathcal{E}\left(\vartheta_{n}\right)=\inf _{\vartheta \geq 0} \mathcal{E}(\vartheta)
$$

We argue that $\lim _{n \rightarrow \infty} \vartheta_{n}=\infty$ is impossible. In fact,

$$
\left\|\left(I+\vartheta_{n} \mathcal{K}\right)^{-1} f-g\right\|_{2}^{2}=\left\|\left(I+\vartheta_{n} \mathcal{K}\right)^{-1} f^{\perp}-g^{\perp}\right\|_{2}^{2}+\left\|\left(I+\vartheta_{n} \mathcal{K}\right)^{-1} f^{N}-g^{N}\right\|_{2}^{2},
$$

and hence, if $\lim _{n \rightarrow \infty} \vartheta_{n}=\infty$, then

$$
\lim _{n \rightarrow \infty}\left\|\left(I+\vartheta_{n} \mathcal{K}\right)^{-1} f-g\right\|_{2}^{2}=\left\|g^{\perp}\right\|_{2}^{2}+\left\|f^{N}-g^{N}\right\|_{2}^{2}
$$

From (3.3), (3.4) and the assumptions on $f^{\perp}$ and $g^{\perp}$ we have

$$
\lim _{n \rightarrow \infty} \mathcal{E}\left(\vartheta_{n}\right)=\left\|g^{\perp}\right\|_{2}^{2}+\left\|f^{N}-g^{N}\right\|_{2}^{2}>\left\|f^{\perp}-g^{\perp}\right\|_{2}^{2}+\left\|f^{N}-g^{N}\right\|_{2}^{2}=\mathcal{E}(0),
$$

which is a contradiction and thus $\left\{\vartheta_{n}\right\}$ is bounded. It follows that there exists a convergent subsequence and an accumulation point $\vartheta^{*} \in[0, \infty)$. 
Since $\vartheta \rightarrow \mathcal{E}(\vartheta)$ is continuous, it follows from (3.3) that every accumulation point is a solution to (3.2).

Now we assume that $\vartheta^{*}=0$ and note that

$$
\mathcal{E}^{\prime}(\vartheta)=-\left\langle(I+\vartheta \mathcal{K})^{-2} \mathcal{K} f,(I+\vartheta \mathcal{K})^{-1} f-g\right\rangle .
$$

We find $\mathcal{E}^{\prime}(0)=-\langle\mathcal{K} f, f-g\rangle$ which by assumption is strictly negative. This contradicts that 0 is a minimum and hence $\vartheta^{*} \in(0, \infty)$.

Remark 3.2. If $K=I$ and $\|f\|_{2}=\|g\|_{2}=1$, then the condition $\langle\mathcal{K} f, f-g\rangle>$ 0 becomes $1>\langle f, g\rangle$ which is equivalent to assuming that $f \neq g$.

We next turn to investigate some of the properties of $\mathcal{E}(\vartheta)$. We shall use that

$$
\mathcal{E}^{\prime \prime}(\vartheta)=3\left\langle(I+\vartheta \mathcal{K})^{-4} \mathcal{K} f, \mathcal{K} f\right\rangle-2\left\langle(I+\vartheta \mathcal{K})^{-3} \mathcal{K} f, \mathcal{K} g\right\rangle .
$$

Since $\mathcal{K} \geq 0$ is symmetric, every element $x \in \mathbb{R}^{n}$ can be expressed as

$$
x=\sum_{i=1}^{r} x_{i} e_{i}+x^{N},
$$

where $\left\{e_{i}\right\}$ are the normalized eigenvectors of $\mathcal{K}$ corresponding to nontrivial eigenvalues $0<\lambda_{1} \leq \cdots \leq \lambda_{r}, r \leq n$ of $\mathcal{K}$. If $r=n$, then $\operatorname{ker}(\mathcal{K})=\{0\}$. We shall express $f^{\perp}=\sum_{i=1}^{r} f_{i} e_{i}$ and $g^{\perp}=\sum_{i=1}^{r} g_{i} e_{i}$.

\subsubsection{The noise-free case}

In this and the following subsections we investigate qualitative properties of $\mathcal{E}(\vartheta)$. We first consider the special case $f^{\perp}=g^{\perp}$, which we refer to as the noise-free case.

Proposition 3.3. Assume that $f^{\perp}=g^{\perp}$ and $f^{\perp} \neq 0$.

(a) Then $\vartheta^{*}=0$ is the unique global solution to (3.2). Moreover, $\vartheta \rightarrow \mathcal{E}(\vartheta)$ is strictly increasing from $\left\|f^{N}-g^{N}\right\|_{2}^{2}$ to $\left\|g^{\perp}\right\|_{2}^{2}+\left\|f^{N}-g^{N}\right\|_{2}^{2}$, it is strictly convex for $\vartheta \in\left[0, \frac{1}{2 \lambda_{r}}\right)$ and concave for $\vartheta \in\left(\frac{1}{2 \lambda_{1}}, \infty\right)$.

(b) If $\lambda_{r} \leq 2 \lambda_{1}$, then there exists a unique $\tilde{\vartheta} \in\left[\frac{1}{2 \lambda_{r}}, \frac{1}{2 \lambda_{1}}\right]$ such that $\mathcal{E}(\vartheta)$ is convex for $\vartheta \in[0, \tilde{\vartheta})$ and concave for $\vartheta \in(\tilde{\vartheta}, \infty)$. 


\section{Proof.}

(a) Note that $\mathcal{E}(0)=\left\|f^{N}-g^{N}\right\|_{2}^{2}$ and

$$
\begin{aligned}
\lim _{\vartheta \rightarrow \infty}\left\|(I+\vartheta \mathcal{K})^{-1} f-g\right\|_{2}^{2} & =\lim _{\vartheta \rightarrow \infty}\left\|(I+\vartheta \mathcal{K})^{-1}\left(f^{N}+f^{\perp}\right)-g\right\|_{2}^{2} \\
& =\left\|g^{\perp}\right\|_{2}^{2}+\left\|f^{N}-g^{N}\right\|_{2}^{2} .
\end{aligned}
$$

By (3.5) and since $f^{\perp}=g^{\perp}$ we have

$$
\mathcal{E}^{\prime}(\vartheta)=-\sum_{i=1}^{r}\left(\frac{\lambda_{i}}{\left(1+\lambda_{i} \vartheta\right)^{3}}-\frac{\lambda_{i}}{\left(1+\lambda_{i} \vartheta\right)^{2}}\right) f_{i}^{2}=\sum_{i=1}^{r} \frac{\lambda_{i}^{2} \vartheta}{\left(1+\lambda_{i} \vartheta\right)^{3}} f_{i}^{2} .
$$

Therefore $\mathcal{E}^{\prime}(0)=0$ and $\mathcal{E}^{\prime}(\vartheta)>0$ for $\vartheta>0$, where we use that $f^{\perp} \neq 0$. Hence $\mathcal{E}$ is strictly increasing from $\left\|f^{N}-g^{N}\right\|_{2}^{2}$ to $\left\|g^{\perp}\right\|_{2}^{2}+\left\|f^{N}-g^{N}\right\|_{2}^{2}$. Similarly we find that

$$
\mathcal{E}^{\prime \prime}(\vartheta)=\sum_{i=1}^{r}\left(\frac{3 \lambda_{i}^{2}}{\left(1+\lambda_{i} \vartheta\right)^{4}}-\frac{2 \lambda_{i}^{2}}{\left(1+\lambda_{i} \vartheta\right)^{3}}\right) f_{i}^{2}=\sum_{i=1}^{r} \frac{\lambda_{i}^{2}}{\left(1+\lambda_{i} \vartheta\right)^{4}}\left(1-2 \lambda_{i} \vartheta\right) f_{i}^{2} .
$$

Hence $\mathcal{E}^{\prime \prime}$ is strictly convex for $\vartheta \in\left[0, \frac{1}{2 \lambda_{m}}\right)$ and strictly concave for $\vartheta \in\left(\frac{1}{2 \lambda_{1}}, \infty\right)$.

(b) We express $\mathcal{E}^{\prime \prime}(\vartheta)=\sum_{i=1}^{r} h_{i}$ where

$$
h_{i}=\frac{\lambda_{i}^{2}}{\left(1+\lambda_{i} \vartheta\right)^{4}}\left(1-2 \lambda_{i} \vartheta\right) f_{i}^{2} \quad \text { and } \quad h_{i}^{\prime}=\frac{-6 \lambda_{i}^{3}}{\left(1+\lambda_{i} \vartheta\right)^{5}}\left(1-\lambda_{i} \vartheta\right) f_{i}^{2} \text {. }
$$

We note that $h_{i}$ is strictly monotonically decreasing on $\left[0, \frac{1}{\lambda_{i}}\right)$ for $i=$ $1, \ldots, r$, and hence $\mathcal{E}^{\prime \prime}$ is strictly decreasing on $\left[0, \frac{1}{\lambda_{r}}\right)$. We have that

$$
\mathcal{E}^{\prime \prime}(\vartheta)>0 \text { for } \vartheta \in\left[0, \frac{1}{2 \lambda_{r}}\right) \text { and } \mathcal{E}^{\prime \prime}(\vartheta)<0 \text { for } \vartheta \in\left(\frac{1}{2 \lambda_{1}}, \infty\right)
$$

Together with $\lambda_{r} \leq 2 \lambda_{1}$ these observations imply the claim. 


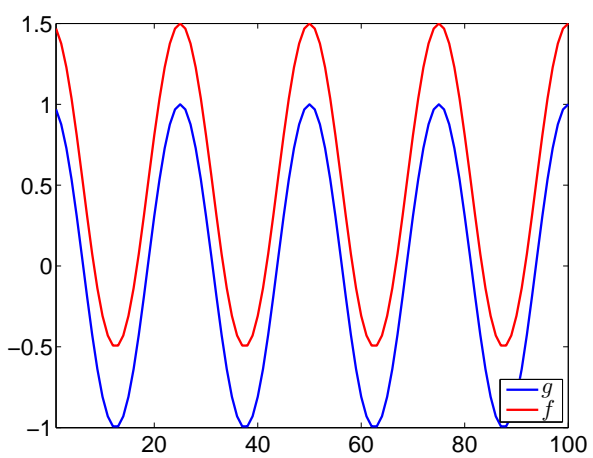

(a)

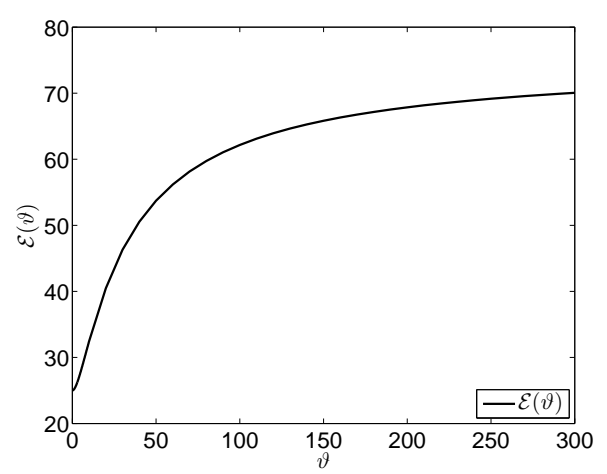

(b)

Figure 2: The noise-free case. (a) shows the discrete cosine signals $g$ and $f$, where we used an offset value of $c=1 / 2$. (b) shows the function values of $\mathcal{E}(\vartheta)$ in dependence of the parameter $\vartheta$.

Example 3.4. Let $g=\left(g_{1}, \ldots, g_{n}\right)$ be a discrete cosine defined by $g_{i}=$ $\cos (8 \pi i / n), 1 \leq i \leq n$ and let $f=\left(f_{1}, \ldots, f_{n}\right)$ be a shifted version computed as $f_{i}=g_{i}+c, c \in \mathbb{R}$. Figure 2 (a) plots the signal $g$ for $n=100$ together with its shifted version $f$, where $c=1 / 2$. Furthermore, let $K$ be a finite differences approximation of a one-dimensional gradient operator, i.e. $(K x)(i)=x(i+1)-x(i)$ if $1 \leq i<n$ and $(K x)(n)=0$. Note that since $(c, \ldots, c)^{T} \in \operatorname{ker}(\mathcal{K}), c \in \mathbb{R}$, we have that $g^{\perp}=f^{\perp}$. The nontrivial eigenvalues of $\mathcal{K}$ are given in ascending order by

$$
\lambda_{i}=4 \sin ^{2}((i \pi) /(2 n)), \quad i=1, \ldots, n-1 .
$$

According to Proposition 3.3 we find that $\mathcal{E}$ is strictly convex for $\vartheta \in[0,0.125)$ and strictly concave for $\vartheta \in(506.648, \infty)$. See also Figure 2.

\subsubsection{The noisy case}

The following result provides sufficient conditions for convexity and concavity of $\mathcal{E}$, for the case where $f^{\perp}$ may differ from $g^{\perp}$.

Proposition 3.5. (convexity/concavity).

(a) If $\|\mathcal{K} g\|_{2}<\frac{3}{2}\|\mathcal{K} f\|_{2}$, then $\mathcal{E}$ is strictly convex on

$$
\left(0, \frac{1}{\|\mathcal{K}\|_{2}}\left(\sqrt{\frac{3}{2} \frac{\|\mathcal{K} f\|_{2}}{\|\mathcal{K} g\|_{2}}}-1\right)\right) .
$$




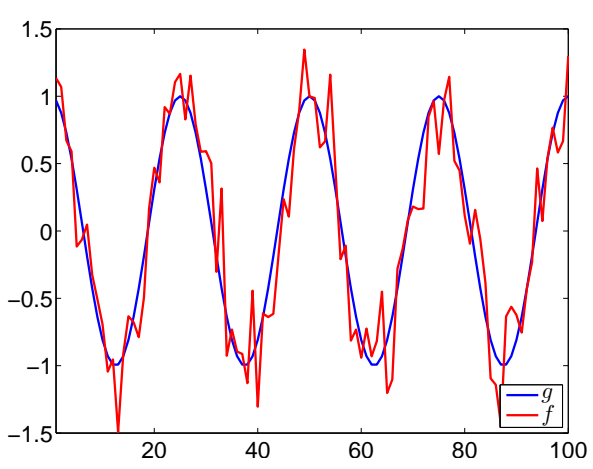

(a)

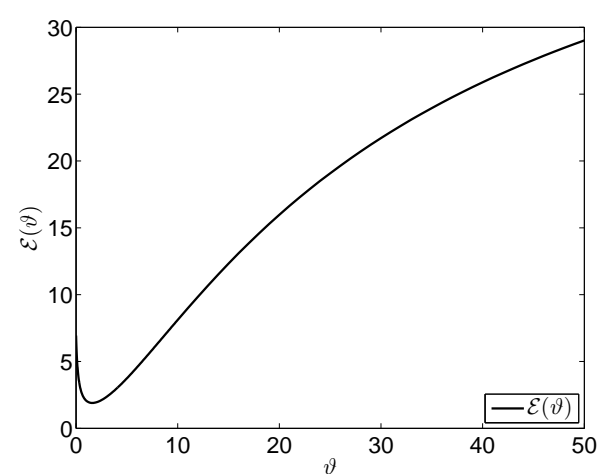

(b)

Figure 3: The noisy case. (a) shows the discrete cosine signals $g$ and its noisy version $f$ with additive Gaussian noise with a standard deviation of $\sigma=1 / 4$. (b) shows the function values of $\mathcal{E}(\vartheta)$ in dependence of the parameter $\vartheta$ together with the bound of strict convexity which is computed according to Proposition 3.5 (a).

(b) If $f^{\perp} \neq 0$, then $\mathcal{E}$ is strictly convex on $(0, \underline{\vartheta})$, where $\underline{\vartheta}=\min _{f_{i} g_{i}>0} \frac{1}{\lambda_{i}}\left(\frac{3 f_{i}^{2}}{2 f_{i} g_{i}}-1\right)$. If $f_{i} g_{i} \leq 0$ for $i=1, \ldots, m$, then $\underline{\vartheta}=\infty$.

(c) If $\sum_{i=1}^{r} \frac{1}{\lambda_{i}^{2}} f_{i} g_{i}>0$, then there exists $\tilde{\vartheta}$ such that $\mathcal{E}$ is strictly concave on $(\tilde{\vartheta}, \infty)$.

Proof.

(a) We have

$$
1=\left\|(I+\vartheta \mathcal{K})^{-1}(I+\vartheta \mathcal{K})\right\|_{2} \leq\left\|(I+\vartheta \mathcal{K})^{-1}\right\|_{2}\|(I+\vartheta \mathcal{K})\|_{2},
$$

from which together with $\|(I+\vartheta \mathcal{K})\|_{2} \leq 1+\vartheta\|\mathcal{K}\|_{2}$ it follows that

$$
\frac{1}{1+\vartheta\|\mathcal{K}\|_{2}} \leq \frac{1}{\|(I+\vartheta \mathcal{K})\|_{2}} \leq\left\|(I+\vartheta \mathcal{K})^{-1}\right\|_{2} \leq 1,
$$

where the upper bound follows from the fact that $(I+\vartheta \mathcal{K})$ is positive 
definite. From (3.6) we have

$$
\begin{array}{r}
\mathcal{E}^{\prime \prime}(\vartheta) \geq\left\|(I+\vartheta \mathcal{K})^{-2} \mathcal{K} f\right\|_{2}\left(3\left\|(I+\vartheta \mathcal{K})^{-2} \mathcal{K} f\right\|_{2}-2\left\|(I+\vartheta \mathcal{K})^{-1} \mathcal{K} g\right\|_{2}\right) \\
\geq\left\|(I+\vartheta \mathcal{K})^{-2} \mathcal{K} f\right\|_{2}\left(\frac{3}{\left(1+\vartheta\|\mathcal{K}\|_{2}\right)^{2}}\|\mathcal{K} f\|_{2}-2\|\mathcal{K} g\|_{2}\right)>0
\end{array}
$$

provided that $\vartheta \in\left(0, \frac{1}{\|\mathcal{K}\|_{2}}\left(\sqrt{\frac{3}{2} \frac{\|\mathcal{K} f\|_{2}}{\|\mathcal{K} g\|_{2}}}-1\right)\right)$.

(b) Let $P=\left\{i \in\{1, \ldots, m\}: f_{i} g_{i}>0\right\}$. Utilizing (3.6) we find

$$
\begin{aligned}
\mathcal{E}^{\prime \prime}(\vartheta) & =3 \sum_{i=1}^{m} \frac{\lambda_{i}^{2}}{\left(1+\vartheta \lambda_{i}\right)^{4}} f_{i}^{2}-2 \sum_{i=1}^{m} \frac{\lambda_{i}^{2}}{\left(1+\vartheta \lambda_{i}\right)^{3}} f_{i} g_{i} \\
& \geq 3 \sum_{i=1, i \notin P}^{m} \frac{\lambda_{i}^{2}}{\left(1+\vartheta \lambda_{i}\right)^{4}} f_{i}^{2}+\sum_{i \in P}^{m} \frac{\lambda_{i}^{2}}{\left(1+\vartheta \lambda_{i}\right)^{4}}\left(3 f_{i}^{2}-2 f_{i} g_{i}\left(1+\vartheta \lambda_{i}\right)\right)>0,
\end{aligned}
$$

for $\vartheta \in(0, \underline{\vartheta})$. Here we also use that $f^{\perp} \neq 0$.

(c) For $\vartheta \geq \frac{1}{\lambda_{1}}$ we have

$$
\mathcal{E}^{\prime \prime}(\vartheta) \leq \frac{3}{\vartheta^{4}} \sum_{i=1}^{r} \frac{1}{\lambda_{i}^{2}} f_{i}^{2}-\frac{2}{\vartheta^{3}} \sum_{i=1}^{r} \frac{\lambda_{i}}{\left(\frac{1}{\vartheta}+\lambda_{i}\right)^{3}} f_{i} g_{i} \leq \frac{3}{\vartheta^{4}} \frac{1}{\lambda_{1}^{2}}\|f\|_{2}^{2}-\frac{1}{4 \vartheta^{3}} \sum_{i=1}^{r} \frac{1}{\lambda_{i}^{2}} f_{i} g_{i}
$$

and the claim follows.

Example 3.6. Let $g$ and $K$ be as defined in Example 3.4, but now let $f$ be a noisy version of $g$, where we added zero-mean Gaussian noise with $\sigma=1 / 4$. Figure 3 (a) plots the cosine signal $g$ for $n=100$ together with its noisy version $f$. According to Proposition 3.5 (a). We get that $\mathcal{E}$ is strictly convex on $\vartheta \in(0, \tilde{\vartheta})$, where $\tilde{\vartheta}=\frac{1}{\|\mathcal{K}\|_{2}}\left(\sqrt{\frac{3}{2} \frac{\|\mathcal{K} f\|_{2}}{\|\mathcal{K} g\|_{2}}}-1\right)$ is computed as $\tilde{\vartheta}=0.8932$. See Figure 3, where the typical quasiconvex behavior of the learning functional $\mathcal{E}$ can be observed.

\subsubsection{A remark on the infinite-dimensional case}

Let $K$ be a closed densely defined linear operator between Hilbert spaces $H$ and $Y$, with $H$ separable. Then $\mathcal{K}=K^{*} K$ is a selfadjoint nonnegative operator in $H$ with dense domain that we denote by $\operatorname{dom}(\mathcal{K})$, see e.g. [18], page 326. Moreover, for every $\lambda$ with $\operatorname{Re} \lambda>0$ the resolvent $(\mathcal{K}+\lambda I)^{-1}$ 
exists as bounded linear operator on $H$, see e.g. [18], page 279. Within this setting we consider for $g \in H, f \in H$ and $\vartheta \geq 0$

$$
\left\{\begin{array}{l}
\min _{\vartheta \geq 0} \mathcal{E}(x(\vartheta))=\|x(\vartheta)-g\|_{H}^{2} \\
\text { subject to } x(\vartheta)=\arg \min _{x} \frac{\vartheta}{2}\|K x\|_{Y}^{2}+\frac{1}{2}\|x-f\|_{H}^{2} .
\end{array}\right.
$$

The necessary and sufficient optimality condition for the lower level problem is given by

$$
(I+\vartheta \mathcal{K}) x=f .
$$

It has a unique solution $x(\vartheta) \in H$ for each $\vartheta \geq 0$. If $\vartheta>0$ then $x(\vartheta) \in$ $\operatorname{dom}(\mathcal{K})$. Again we have an equivalent reduced problem

$$
\min _{\vartheta \geq 0} \mathcal{E}(\vartheta)=\left\|(I+\vartheta \mathcal{K})^{-1} f-g\right\|_{H}^{2}
$$

and the orthogonal decomposition

$$
x=x^{N}+x^{\perp} \in \operatorname{ker}(\mathcal{K}) \oplus \overline{\operatorname{ran}(\mathcal{K})},
$$

where the closure is taken in $H$. We assume that $(I+\vartheta \mathcal{K})^{-1}$ is compact for some (or equivalent all) $\vartheta>0$. Then the spectrum of $\mathcal{K}$ consists entirely of isolated eigenvalues $0<\lambda_{1} \leq \lambda_{2} \ldots$ of finite multiplicity plus possibly the

eigenvalue 0 , and every $x \in H$ can be expressed as $x=\sum_{i=1}^{\infty} x_{i} e_{i}+x^{N}$ with $x^{N} \in \operatorname{ker}(\mathcal{K})$ and $e_{i}$ eigenvectors of $\mathcal{K}$, associated to the eigenvalues $\neq 0$. We have the analogue of Proposition 3.1.

Proposition 3.7. If $\left\|f^{\perp}-g^{\perp}\right\|_{H}<\left\|g^{\perp}\right\|_{H}$, then (3.10) admits a solution $\vartheta^{*} \geq 0$. If moreover, $f \in \operatorname{dom}(\mathcal{K})$ and $\langle\mathcal{K} f, f-g\rangle_{H}>0$, then $\vartheta^{*}>0$.

Proof. Using the fact that $(I+\vartheta \mathcal{K})^{-1}$ leaves $\operatorname{ker}(\mathcal{K})$ and $\left(\operatorname{ker}(\mathcal{K})^{\perp}\right.$ invariant we can proceed as in the proof of Proposition 3.1 to get the first part of the result. Note that $\lim _{\vartheta \rightarrow 0^{+}}(I+\vartheta \mathcal{K}) v=v$ for all $v \in H$. Consequently $\mathcal{E}^{\prime}(\vartheta)$ is continuous on $[0, \infty)$ if $f \in \operatorname{dom}(\mathcal{K})$. The proof of the second part now follows as the one of Proposition 3.1.

\subsection{Multiple priors}

In this section we study the $\ell_{2}$ model with multiple priors, i.e. $p=2$ and $q \geq 1$. It is defined as

$$
\min _{x \in \mathbb{R}^{n}} \frac{1}{2} \sum_{k=1}^{q} \vartheta_{k}\left\|K_{k} x\right\|_{2}^{2}+\frac{1}{2}\|x-f\|_{2}^{2},
$$


with the parameter vector $\vartheta=\left(\vartheta_{1}, \ldots, \vartheta_{q}\right) \geq 0$. The minimum of the above problems is characterized by

$$
x+\sum_{k=1}^{q} \vartheta_{k} \mathcal{K}_{k} x=f,
$$

or equivalently $x=\left(I+\sum_{k=1}^{q} \vartheta_{k} \mathcal{K}_{k}\right)^{-1} f$. The reduced quadratic learning functional is then given by

$$
\min _{\vartheta \geq 0} \mathcal{E}(\vartheta)=\frac{1}{2}\left\|\left(I+\sum_{k=1}^{q} \vartheta_{k} \mathcal{K}_{k}\right)^{-1} f-g\right\|_{2}^{2} .
$$

For convenience we introduce the symmetric positive definite matrix

$$
\mathcal{R}=\left(I+\sum_{k=1}^{q} \vartheta_{k} \mathcal{K}_{k}\right)^{-1} .
$$

To guarantee existence the following condition will be used

$$
\inf \left\{\|\tilde{x}-g\|_{2}: \tilde{x} \in \operatorname{ker}\left(K_{k}\right) \text { for some } k=1, \ldots, q\right\}>\|f-g\|_{2} \text {. }
$$

We observe that in case $\operatorname{ker}\left(K_{k}\right)=\{0\}$ for all $k$, condition (3.13) amounts to $\|g\|_{2}>\|f-g\|_{2}$. If $q=1$, then (3.13) is equivalent to assuming that $\left\|g^{\perp}\right\|_{2}>\left\|f^{\perp}-g^{\perp}\right\|_{2}$. This condition was already used for the singleparameter case in Proposition 3.1.

Proposition 3.8. If (3.13) holds and $\operatorname{ker}\left(K_{k}\right) \cap \operatorname{ker}\left(K_{l}\right)=\{0\}$ for all $k \neq l$, then (3.12) admits a solution.

Proof. Let $\left\{\vartheta^{n}\right\}_{n=1}^{\infty}$ denote a minimizing sequence and suppose that $\lim _{n \rightarrow \infty}\left\|\vartheta^{n}\right\|_{2}=$ $\infty$. Then there exist index sets $\mathcal{J} \subseteq\{1, \ldots, q\}, \overline{\mathcal{J}}=\{1, \ldots, q\} \backslash \mathcal{J}$ and a constant $\kappa_{1}$ such that

$$
\lim _{n \rightarrow \infty} \vartheta_{k}^{n}=\infty \text { for } k \in \mathcal{J}, \text { and }\left|\vartheta_{k}^{n}\right| \leq \kappa_{1} \text { for } k \in \overline{\mathcal{J}} \text { and all } n \text {. }
$$

We set

$$
x^{n}=\left(I+\sum_{k=1}^{q} \vartheta_{k}^{n} \mathcal{K}_{k}\right)^{-1} f .
$$


Clearly $\left\{x^{n}\right\}$ is bounded and hence on a subsequence, denoted by the same index, $\lim _{n \rightarrow \infty} x^{n}=\hat{x}$ for some $\hat{x} \in \mathbb{R}^{n}$. From $(3.15)$

$$
\sum_{k \in \mathcal{J}} \vartheta_{k}^{n} \mathcal{K}_{k} x^{n}=f-\left(x_{n}+\sum_{k \in \overline{\mathcal{J}}} \vartheta_{k}^{n} \mathcal{K}_{k} x^{n}\right)
$$

Taking the inner product with $x^{n}$ and observing that the righthand side is bounded

$$
\min _{k \in \mathcal{J}} \vartheta_{k}^{n} \sum_{k \in \mathcal{J}}\left\|K_{k} x^{n}\right\|_{2}^{2} \leq \kappa_{2}
$$

for a constant $\kappa_{2}$ independent of $n$. Since $\min _{k \in \mathcal{J}} \vartheta_{k}^{n} \rightarrow \infty$ for $n \rightarrow \infty$ we find $\lim _{n \rightarrow \infty} K_{k} x^{n}=K_{k} \hat{x}=0$ for all , meaning that $\hat{x} \in \operatorname{ker}\left(K_{k}\right)$ for all $k \in \mathcal{J}$. Since $\left\{\vartheta^{n}\right\}$ was chosen as minimizing sequence we obtain

$$
\inf _{\vartheta \geq 0} \mathcal{E}(\vartheta)=\lim _{n \rightarrow \infty}\left\|x^{n}-g\right\|_{2}^{2}=\|\hat{x}-g\|_{2}^{2}>\|f-g\|_{2}^{2}=\mathcal{E}(0)
$$

where we used (3.13). This is a contradiction and hence every minimizing sequence is bounded. Since $\vartheta \rightarrow \mathcal{E}(\vartheta)$ is continuous the claim follows.

The partial derivatives of $\mathcal{E}$ with respect to $\vartheta_{k}$ are given by

$$
(\nabla \mathcal{E}(\vartheta))_{k}=-\left\langle\mathcal{R} f-g, \mathcal{R} \mathcal{K}_{k} \mathcal{R} f\right\rangle \text { for } k=1, \ldots, q,
$$

where $\mathcal{R}$ is evaluated at $\vartheta$. Taking into account the inequality constraint $\vartheta \geq 0$ in (3.12), the first order necessary condition is given by

$$
\nabla \mathcal{E}\left(\vartheta^{*}\right)-\mu=0, \mu \geq 0, \vartheta^{*} \geq 0,\left\langle\mu, \vartheta^{*}\right\rangle=0
$$

where $\mu \in \mathbb{R}^{q}$ is the Lagrange multiplier associated to the constraint $\vartheta \geq 0$. It can be checked that the three last conditions can be equivalently expressed as

$$
\mu-\max (0, \mu-c \vartheta)=0 .
$$

For the Hessian of $\mathcal{E}$ we obtain for $k=1, \ldots, q$ and $l=1, \ldots, q$ the expression

$$
\nabla^{2} \mathcal{E}(\vartheta)=M_{1}+M_{2}
$$

where

$$
\left(M_{1}\right)_{k, l}=\left\langle\mathcal{R} \mathcal{K}_{k} \mathcal{R} f, \mathcal{R} \mathcal{K}_{l} \mathcal{R} f\right\rangle \text { and }\left(M_{2}\right)_{k, l}=\left\langle\mathcal{R} f-g, \mathcal{R} \mathcal{K}_{k} \mathcal{R} \mathcal{K}_{l} \mathcal{R} f+\mathcal{R} \mathcal{K}_{l} \mathcal{R} \mathcal{K}_{k} \mathcal{R} f\right\rangle
$$

are symmetric matrices. 
Let $\mathcal{A}=\left\{k \in\{1, \ldots, q\}:\left(\vartheta^{*}\right)_{k}=0\right\}$ denote the set of active constraints for some local solution $\vartheta^{*}$ of (3.11). Then, the second order necessary optimality condition implies that

$$
\nabla^{2} \mathcal{E}\left(\vartheta^{*}\right) \text { is semidefinite on } T,
$$

where $T$ is the tangent space of the active constraints $T=\left\{\vartheta \in \mathbb{R}^{q}: \vartheta_{k}=\right.$ 0 for all $k \in \mathcal{A}\}$. Note that $M_{1}$ is a Gram-matrix corresponding to the vectors $\left\{\mathcal{R} \mathcal{K}_{k} \mathcal{R} f\right\}_{k=1}^{q}$. We assume that

$$
\left\{\mathcal{K}_{k} y\right\}_{k=1}^{q} \text { is linearly independent for any } y \in \mathbb{R}^{n} \text {. }
$$

Then, with $y=\mathcal{R} f$ and since $\mathcal{R}$ is positive definite, $\left\{\mathcal{R} \mathcal{K}_{k} \mathcal{R} f\right\}_{k=1}^{m}$ is linearly independent and $M_{1}$ is nonsingular. If $\|\mathcal{R} f-g\|_{2}$ is sufficiently small, then $M_{1}+M_{2}$ is nonsingular as well. This implies that $\nabla^{2} \mathcal{E}\left(\vartheta^{*}\right)>0$ on $\mathbb{R}^{q}$. We summarize our discussion in a theorem.

Theorem 3.9. Assume that (3.18) is satisfied and let $\vartheta^{*}$ be a local solution of (3.11). Then, if

$$
\left\|\left(I+\sum_{k=1}^{q} \vartheta_{k}^{*} \mathcal{K}_{k}\right)^{-1} f-g\right\|_{2} \text { is sufficiently small, }
$$

the second order sufficient optimality condition is satisfied at $\vartheta^{*}$, in particular, $\vartheta^{*}$ is a locally unique minimum.

Note that for $f=g$ we have $\vartheta^{*}=0$ as global solution. Therefore (3.19) can be interpreted as smallness condition on the error in the data.

\subsection{Newton algorithm}

We propose and analyse a semi-smooth Newton scheme to solve (3.12). For this purpose we express the necessary optimality condition (3.16) in the form

$$
\left\{\begin{array}{l}
\nabla \mathcal{E}\left(\vartheta^{*}\right)-\mu=0 \\
\mu-\max (0, \mu-c \vartheta)=0
\end{array}\right.
$$

where $c>0$ is an arbitrarily fixed constant. To solve (3.20) we utilize a semi-smooth Newton algorithm which is outlined in Algorithm 3.1. To analyze this algorithm the vectors $\delta \vartheta$ and $\delta \mu$ are decomposed into inactive 
Algorithm 3.1 Newton Learning for $\ell_{2}\left(\mathrm{NL}-\ell_{2}\right)$

(i) Choose $\left(\vartheta^{0}, \mu^{0}\right) \in \mathbb{R}^{r} \times \mathbb{R}^{q}$, set $n=0$

(ii) Determine $\mathcal{A}^{n}=\left\{k: \mu_{k}^{n}-c \vartheta_{k}^{n} \geq 0\right\}, \mathcal{I}^{n}=\left\{k: \mu_{k}^{n}-c \vartheta_{k}^{n}<0\right\}$

(iii) Assign $M=\nabla^{2} \mathcal{E}\left(\vartheta^{n}\right), P=\operatorname{diag}\left(p_{k}\right), Q=\operatorname{diag}\left(q_{k}\right)$ where

$$
p_{k}=\left\{\begin{array}{l}
c \text { if } k \in \mathcal{A}^{n} \\
0 \text { if } k \in \mathcal{I}^{n}
\end{array} \quad q_{k}=\left\{\begin{array}{l}
0 \text { if } k \in \mathcal{A}^{n} \\
1 \text { if } k \in \mathcal{I}^{n} .
\end{array}\right.\right.
$$

(iv) Solve for $(\delta \vartheta, \delta \mu)$

$$
\left(\begin{array}{cc}
M & -I \\
P & Q
\end{array}\right)\left(\begin{array}{l}
\delta \vartheta \\
\delta \mu
\end{array}\right)=-\left(\begin{array}{c}
\nabla \mathcal{E}\left(\vartheta^{n}\right)-\mu^{n} \\
\mu^{n}-\max \left(0, \mu^{n}-c \vartheta^{n}\right)
\end{array}\right)
$$

(v) $\left(\vartheta^{n+1}, \mu^{n+1}\right)=\left(\vartheta^{n}, \mu^{n}\right)+(\delta \vartheta, \delta \mu)$, set $n=n+1$ and goto (ii).

and active components $(\delta \vartheta)_{\mathcal{I}},(\delta \vartheta)_{\mathcal{A}}$ and $(\delta \mu)_{\mathcal{I}},(\delta \mu)_{\mathcal{A}}$ respectively, and $M$ is partitioned accordingly

$$
M=\left(\begin{array}{ll}
M_{\mathcal{I I}} & M_{\mathcal{I} \mathcal{A}} \\
M_{\mathcal{A I}} & M_{\mathcal{A} \mathcal{A}}
\end{array}\right)
$$

Here, for notational convenience the unknowns are ordered in such a manner that the inactive coordinates appear first and the active ones last, and the iteration index for the sets $\mathcal{A}^{n}$ and $\mathcal{I}^{n}$ is dropped. From the second equation in (3.21) we obtain

$$
(\delta \vartheta)_{\mathcal{A}}=-\vartheta_{\mathcal{A}}^{n}, \quad(\delta \mu)_{\mathcal{I}}=-\mu_{\mathcal{I}}^{n}, \quad \vartheta_{\mathcal{A}}^{n+1}=0, \quad \mu_{\mathcal{I}}^{n+1}=0 .
$$

Turning to the first equation in (3.21) we first solve for the inactive components of $\delta \vartheta$ by

$$
M_{\mathcal{I I}}(\delta \vartheta)_{\mathcal{I}}=-M_{\mathcal{I} \mathcal{A}}(\delta \vartheta)_{\mathcal{A}}-\left(\nabla \mathcal{E}\left(\vartheta^{n}\right)\right)_{\mathcal{I}}
$$

and then assign

$$
(\delta \mu)_{\mathcal{A}}=M_{\mathcal{A I}}(\delta \vartheta)_{\mathcal{I}}+M_{\mathcal{A} \mathcal{A}}(\delta \vartheta)_{\mathcal{A}}+\left(\nabla \mathcal{E}\left(\vartheta^{n}\right)\right)_{\mathcal{A}}-\mu_{\mathcal{A}}^{n}
$$

Note that while (3.21) is asymmetric, system (3.23) which is of the dimension of the inactive set, is symmetric. 
Theorem 3.10. Let $\vartheta^{*}$ be a local solution of (3.12) with associated Lagrange multiplier $\mu^{*}$, and suppose that (3.18) and (3.19) hold. Then, if $\left\|\left(\vartheta^{0}, \mu^{0}\right)-\left(\vartheta^{*}, \mu^{*}\right)\right\|_{2}$ is sufficiently small, the iterations of Algorithm 3.1 converge superlinearly to $\left(\vartheta^{*}, \mu^{*}\right)$.

Proof. We verify here the requirements for superlinear convergence of the semi-smooth Newton method as given in e.g. [17],pg.238. The max-operation is well-known to be semi-smooth, see e.g. $[17,30]$ and the references given there and $D \max (0, x)=\chi_{\{x \geq 0\}}$ is a generalized or Newton derivative. Here $\left(\chi_{\{x \geq 0\}}\right)_{i}=1$ if $x_{i} \geq 0$ and $\left(\chi_{\{x \geq 0\}}\right)_{i}=0$ otherwise. This choice of generalized derivative determines step (iii) of Algorithm 3.1. The proof will be finished, if we argue that the system matrices

$$
H(\vartheta, \mu)=\left(\begin{array}{cc}
M(\vartheta) & -I \\
P(\vartheta, \mu) & Q(\vartheta, \mu)
\end{array}\right)
$$

are invertible with uniformly bounded inverses in a neighborhood $B_{\rho}\left(\vartheta^{*}, \mu^{*}\right)$ of $\left(\vartheta^{*}, \mu^{*}\right)$ for some radius $\rho>0$. The notation $H(\vartheta, \mu)$ emphasizes the dependence of $M, P$, and $Q$ on $\vartheta$ and $\mu$. The discussion before Theorem 3.10 implies that $\nabla^{2} \mathcal{E}\left(\vartheta^{*}\right)=M\left(\vartheta^{*}\right)>0$. Hence there exists a neighborhood $B_{\rho}\left(\vartheta^{*}\right)$, with $\rho>0$, and $\kappa>0$ such that $\left\|M^{-1}(\vartheta)\right\|_{2} \leq \kappa$ for all $\vartheta \in B_{\rho}\left(\vartheta^{*}\right)$. In particular, this implies that $\left\|M(\vartheta)_{\mathcal{I} \mathcal{I}}^{-1}\right\|_{2} \leq \kappa$ for all $\vartheta \in B_{\rho}\left(\vartheta^{*}\right)$ and any combination of $\mathcal{I} \in\{1, \ldots, q\}$. Now consider for $\vartheta \in B_{\rho}\left(\vartheta^{*}\right), \mu \in \mathbb{R}^{q}$ and $(y, z) \in \mathbb{R}^{2 q}$

$$
H(\vartheta, \mu)\left(\begin{array}{l}
\delta \vartheta \\
\delta \mu
\end{array}\right)=\left(\begin{array}{l}
y \\
z
\end{array}\right)
$$

As in the computation before the statement of the theorem we find

$$
(\delta \vartheta)_{\mathcal{A}}=\frac{1}{c} z_{\mathcal{A}},(\delta \mu)_{\mathcal{I}}=z_{\mathcal{I}}
$$

From the first equation in (3.24) we find

$$
\begin{array}{r}
M_{\mathcal{I} \mathcal{I}}(\vartheta)(\delta \vartheta)_{\mathcal{I}}=-\frac{1}{c} M_{\mathcal{I} \mathcal{A}}(\vartheta) z_{\mathcal{A}}+z_{\mathcal{I}}+y_{\mathcal{I}} \\
(\delta \mu)_{\mathcal{A}}=M_{\mathcal{A I}}(\delta \vartheta)_{\mathcal{I}}+M_{\mathcal{A \mathcal { A }}}(\delta \vartheta)_{\mathcal{A}}-y_{\mathcal{A}}
\end{array}
$$

Combining these equalities, invertibility of $H(\vartheta, \mu)$ with uniformly bounded inverses for $(\vartheta, \mu)$ in a neighborhood of $\left(\vartheta^{*}, \mu^{*}\right)$ follows. 


\section{The $\ell_{1}$ model}

In this section we analyse variational models with $\ell_{1}$ and hence non-differentiable regularization terms. This type of model has great impact in signal processing, in particular in imaging and compressed sensing.

\subsection{Problem formulation and existence}

$$
\left\{\begin{array}{l}
\min _{\vartheta \geq 0} \mathcal{E}(x(\vartheta))=\|x(\vartheta)-g\|_{2}^{2} \\
\text { subject to } x(\vartheta)=\arg \min _{x} \sum_{k=1}^{q} \vartheta_{k}\left\|K_{k} x\right\|_{1}+\frac{1}{2}\|x-f\|_{2}^{2} .
\end{array}\right.
$$

The lower level problem (4.1) admits a unique solution $x=x(\vartheta)$. Its optimality condition is given by

$$
\left\{\begin{array}{l}
\sum_{k=1}^{q} \vartheta_{k} K_{k}^{*} \lambda^{k}+x=f \\
\lambda_{i}^{k} \in\left\{\begin{array}{r}
\operatorname{sgn}\left(K_{k} x\right)_{i} \text { if }\left(K_{k} x\right)_{i} \neq 0 \\
{[-1,1] \text { if }\left(K_{k} x\right)_{i}=0 .}
\end{array}\right.
\end{array}\right.
$$

We have the following existence result analogous to Proposition 3.1.

Proposition 4.1. If (3.13) holds, then (4.1) admits a solution $\vartheta^{*} \geq 0$.

Proof. We first argue that $\vartheta \rightarrow x(\vartheta)$, with $x(\vartheta)$ the solution to the lower level problem, is continuous. Let $\vartheta^{n} \rightarrow \bar{\vartheta}$ and $x_{n}=x\left(\vartheta^{n}\right)$. Since

$$
\sum_{k=1}^{q} \vartheta_{k}^{n}\left\|K_{k} x_{n}\right\|_{1}+\frac{1}{2}\left\|x_{n}-f\right\|_{2}^{2} \leq \frac{1}{2}\|f\|_{2}^{2}
$$

the sequence $\left\{x_{n}\right\}$ is bounded and hence it admits a convergent subsequence $x_{n_{k}} \rightarrow \bar{x}$. We need to argue that $\bar{x}=x(\bar{\vartheta})$. For this purpose we note that

$\sum_{k=1}^{q} \vartheta_{k}^{n}\left\|K_{k} x_{n}\right\|_{1}+\frac{1}{2}\left\|x_{n}-f\right\|_{2}^{2} \leq \sum_{k=1}^{q} \vartheta_{k}^{n}\left\|K_{k} x\right\|_{1}+\frac{1}{2}\|x-f\|_{2}^{2}$ for all $x \in \mathbb{R}^{n}$

implies that

$$
\sum_{k=1}^{q} \overline{\vartheta_{k}}\left\|K_{k} \bar{x}\right\|_{1}+\frac{1}{2}\|\bar{x}-f\|_{2}^{2} \leq \sum_{k=1}^{q} \overline{\vartheta_{k}}\left\|K_{k} x\right\|_{1}+\frac{1}{2}\|x-f\|_{2}^{2} \text { for all } x \in \mathbb{R}^{n},
$$


and hence $\bar{x}=x(\bar{\vartheta})$, since the solution to the lower level problem is unique. Next, let $\left\{\vartheta^{n}\right\}_{n=1}^{\infty}$ be a minimizing sequence and abbreviate $x_{n}=x\left(\vartheta^{n}\right)$. If $\lim _{n \rightarrow \infty}\left\|\vartheta^{n}\right\|_{2}=\infty$ determine $\mathcal{J}$ as in (3.14). Since

$$
\sum_{k=1}^{r} \vartheta_{k}^{n}\left\|K_{k} x_{n}\right\|_{1}+\frac{1}{2}\left\|x_{n}-f\right\|_{2}^{2} \leq \frac{1}{2}\|f\|_{2}^{2}
$$

we deduce that $\left\{x_{n}\right\}_{n=1}^{\infty}$ is bounded and that $\lim _{n \rightarrow \infty}\left\|K_{i} x_{n}\right\|_{1}=0$ for all $i \in \mathcal{J}$. Hence there exists a subsequence, denoted by the same symbol, and $\hat{x}$ such that $\lim _{n \rightarrow \infty} x_{n}=\hat{x}$ and $K_{i} \hat{x}=0$ for all $i \in \mathcal{J}$. In particular, $\hat{x}$ is contained in the kernel of at least one operator $K_{i}$ and thus by (3.13)

$$
\inf _{\vartheta \geq 0} \mathcal{E}(x(\vartheta))=\lim _{n \rightarrow \infty} \mathcal{E}\left(x_{n}\right)=\lim _{n \rightarrow \infty}\left\|x_{n}-g\right\|_{2}^{2}=\|\hat{x}-g\|_{2}^{2}<\|f-g\|_{2}^{2}=\mathcal{E}(x(0)) \text {, }
$$

which contradicts the choice of $\left\{\vartheta_{n}\right\}_{n=1}^{\infty}$ as minimizing sequence. Hence $\left\{\vartheta_{n}\right\}_{n=1}^{\infty}$ is bounded in $\mathbb{R}^{q}$. Consequently there exists another subsequence denoted by the same symbol, and $\vartheta^{*} \in[0, \infty)$ such that $\lim _{n \rightarrow \infty} \vartheta_{n}=\vartheta^{*}$. Since $\vartheta \rightarrow x(\vartheta)$, and hence $\vartheta \rightarrow \mathcal{E}(x(\vartheta))$ are continuous, it follows that every accumulation point $\vartheta^{*}$ of $\left\{\vartheta_{n}\right\}$ is a solution to (4.1), and $x^{*}=x\left(\vartheta^{*}\right)$.

Remark 4.2. In case of only one prior, we can give a sufficient condition to exclude the case that $\vartheta^{*}=0$. For this purpose we assume that

$$
(K g)_{i}=0 \text { if }(K f)_{i}=0 \text { and }\left\langle K f-g, \frac{K f}{|K f|}\right\rangle>0,
$$

where $\frac{K f}{|K f|}$ is interpreted componentwise as $\frac{(K f)_{i}}{\left|(K f)_{i}\right|}$, if $(K f)_{i} \neq 0$ and $\frac{(K f)_{i}}{\left|(K f)_{i}\right|}$ is interpreted as some element in $[-1,1]$, if $(K f)_{i}=0$. We now exclude that $\vartheta^{*}=0$ is the minimum. For this purpose we argue that $\left.\frac{d}{d \vartheta} \mathcal{E}(x(\vartheta))\right|_{\vartheta=0^{+}}$ exists and is negative. We have

$$
\begin{aligned}
\mathcal{E}(x(\vartheta))-\mathcal{E}(x(0)) & =\langle x(\vartheta)+x(0)-2 g, x(\vartheta)-x(0)\rangle \\
& =-\vartheta\left\langle x(\vartheta)+f-2, K^{*} \lambda(\vartheta)\right\rangle=-\vartheta\langle K(x(\vartheta)+f-2 g), \lambda(\vartheta)\rangle,
\end{aligned}
$$

where we use that $x(0)=f$.

Let $\mathcal{I}=\left\{i:(K x(0))_{i} \neq 0\right\}$. Then $(K x(\vartheta))_{i} \neq 0, \quad$ for all $i \in \mathcal{I}$ and all $\vartheta>0$ sufficiently small. For these $i$ and $\vartheta$ we have

$$
\lambda_{i}(\vartheta)=\frac{(K x(\vartheta))_{i}}{\left|(K x(\vartheta))_{i}\right|} \rightarrow \frac{(K x(0))_{i}}{\left|(K x(0))_{i}\right|} .
$$


For $i \notin \mathcal{I}$ we have $\lambda_{i}(\vartheta) \in[-1,1]$ and $(K(x(\vartheta)+f-2 g))_{i} \rightarrow 0$ for $\vartheta \rightarrow 0^{+}$, where we use that $\lim _{\vartheta \rightarrow 0^{+}} x(\vartheta)_{i}=f_{i}$ and (4.3). Therefore

$$
\lim _{\vartheta \rightarrow 0^{+}} \frac{1}{\vartheta}(\mathcal{E}(x(\vartheta))-\mathcal{E}(x(0)))=-2\left\langle K(f-g), \frac{K f}{|K f|}\right\rangle,
$$

and $\vartheta \rightarrow \mathcal{E}(x(\vartheta))$ is differentiable at $\vartheta=0^{+}$. By $(4.3)$ we have $\left.\frac{d}{d t} \mathcal{E}(x(\vartheta))\right|_{\vartheta=0^{+}}<$ 0 and hence $\vartheta=0$ cannot be a solution to (4.1). We note that the condition $\left\langle K(f-g), \frac{K f}{|K f|}\right\rangle>0$ can equally well be expressed as $\langle K(f-g), \lambda(0)\rangle>0$ for any Lagrange multiplier $\lambda(0)$ associated to $\vartheta=0$.

\subsection{Optimality system}

To derive an optimality system for (4.1) we use a regularization approach and consider

$$
\left\{\begin{array}{l}
\min _{\vartheta \geq 0} \mathcal{E}(x(\vartheta))=\|x(\vartheta)-g\|_{2}^{2} \\
\text { subject to } x(\vartheta)=\arg \min _{x} \sum_{k=1}^{q} \vartheta_{k} \sum_{j=1}^{m} n_{\varepsilon}\left(\left(K_{k} x\right)_{j}\right)+\frac{1}{2}\|x-f\|_{2}^{2},
\end{array}\right.
$$

where, for $\varepsilon>0$,

$$
n_{\varepsilon}(t)=\left\{\begin{array}{cc}
-\frac{1}{8 \varepsilon^{3}} t^{4}+\frac{3}{4 \varepsilon} t^{2}+\frac{3 \varepsilon}{8} & \text { if }|t|<\varepsilon \\
|t| & \text { else } .
\end{array}\right.
$$

The following properties of $n_{\varepsilon}$ will be used repeatedly

$$
\left\{\begin{array}{l}
n_{\varepsilon} \in C^{2}(\mathbb{R}, \mathbb{R}), n_{\varepsilon}( \pm \varepsilon)= \pm \varepsilon, n_{\varepsilon}^{\prime}( \pm \varepsilon)= \pm 1, n_{\varepsilon}^{\prime \prime}( \pm \varepsilon)=0, n_{\varepsilon}^{\prime}(t) \in[-1,1] \\
n_{\varepsilon}^{\prime \prime}(t) \in\left[0, \frac{3}{2 \varepsilon}\right], n_{\varepsilon}(t) \geq t \text { for all } t \in \mathbb{R} .
\end{array}\right.
$$

Furthermore, we have

$$
\begin{aligned}
& n_{\varepsilon}^{\prime}(t)=\left\{\begin{array}{rl}
-\frac{1}{2 \varepsilon^{3}} t^{3}+\frac{3}{2 \varepsilon} t & \text { if } \\
\operatorname{sgn}(t) & \text { else }
\end{array}|t|<\varepsilon\right. \\
& n_{\varepsilon}^{\prime \prime}(t)=\left\{\begin{array}{rll}
-\frac{3}{2 \varepsilon^{3}} t^{2}+\frac{3}{2 \varepsilon} & \text { if } & |t|<\varepsilon \\
0 & \text { else } &
\end{array}\right.
\end{aligned}
$$




$$
n_{\varepsilon}^{\prime \prime \prime}(t)=\left\{\begin{array}{rll}
-\frac{3}{\varepsilon^{3}} t & \text { if } & |t|<\varepsilon \\
\left\{0, \frac{3}{\varepsilon^{2}}\right\} & \text { if } & t=-\varepsilon \\
\left\{-\frac{3}{\varepsilon^{2}}, 0\right\} & \text { if } & t=\varepsilon \\
0 & \text { else. } &
\end{array}\right.
$$

At $t= \pm \varepsilon$ we consider, for the time being, the third derivative to be multivalued consisting of the right and left directional derivatives. It is simple to argue the existence of a unique lower-level solution $x_{\varepsilon}(\vartheta)$ for each $\varepsilon>0$. It is characterized as the solution $x=x(\vartheta)$ to

$$
x+\sum_{k=1}^{q} \vartheta_{k} K_{k}^{T} N_{\varepsilon}^{\prime}\left(K_{k} x\right)=f
$$

where

$$
N_{\varepsilon}^{\prime}\left(K_{k} x\right)=\left(n_{\varepsilon}^{\prime}\left(\left(K_{k} x\right)_{1}\right), \ldots, n_{\varepsilon}^{\prime}\left(\left(K_{k} x\right)_{m}\right)\right)^{T} \in \mathbb{R}^{m} .
$$

Since $t \rightarrow n_{\varepsilon}^{\prime}(t)$ is monotone, the operator $x \rightarrow x+\sum_{k=1}^{q} \vartheta_{k} K_{k}^{T} N_{\varepsilon}^{\prime}\left(K_{k} x\right)$ is strictly monotone and hence the solution to (4.7) is unique. Using (4.7) it follows that $\vartheta \rightarrow x_{\varepsilon}(\vartheta)$ is differentiable on $[0, \infty)^{q}$ for each $\varepsilon>0$, with the sensitivity equation given by

$$
D_{\vartheta} x+\left[K_{k}^{T} N_{\varepsilon}^{\prime}\left(K_{k} x\right)\right]+\sum_{k=1}^{q} \vartheta_{k} K_{k}^{T} N_{\varepsilon}^{\prime \prime}\left(K_{k} x\right) K_{k} D_{\vartheta} x=0
$$

where

$$
D_{\vartheta} x \in \mathbb{R}^{n \times q},\left[K_{k}^{T} N_{\varepsilon}^{\prime}\left(K_{k} x\right)\right]=\left(K_{1}^{T} N_{\varepsilon}^{\prime}\left(K_{1} x\right), \ldots, K_{q}^{T} N_{\varepsilon}^{\prime}\left(K_{q} x\right)\right) \in \mathbb{R}^{n \times q}
$$

and

$$
N_{\varepsilon}^{\prime \prime}\left(K_{k} x\right)=\operatorname{diag}\left(n_{\varepsilon}^{\prime \prime}\left(\left(K_{k} x\right)_{1}\right), \ldots, n_{\varepsilon}^{\prime \prime}\left(\left(K_{k} x\right)_{m}\right)\right) \in \mathbb{R}^{m \times m} .
$$

Let $\vartheta_{\varepsilon}$ denote a solution to (4.4), which exists under the assumption of Proposition 4.1. Then the first order optimality condition for (4.4) is given by

$$
D_{\vartheta} \mathcal{E}\left(x_{\varepsilon}\left(\vartheta_{\varepsilon}\right)\right)\left(\vartheta-\vartheta_{\varepsilon}\right)=2\left\langle x_{\varepsilon}\left(\vartheta_{\varepsilon}\right)-g, D_{\vartheta} x\left(\vartheta_{\varepsilon}\right)\left(\vartheta-\vartheta_{\varepsilon}\right)\right\rangle \geq 0 \text {, for all } \vartheta \geq 0 \text {. }
$$

To eliminate $D_{\vartheta} x_{\varepsilon}$ from the first order condition (4.9) we introduce the adjoint equation

$$
p+\sum_{k=1}^{q} \vartheta_{k} K_{k}^{T} N_{\varepsilon}^{\prime \prime}\left(K_{k} x\right) K_{k} p=-\left(x_{\varepsilon}\left(\vartheta_{\varepsilon}\right)-g\right) .
$$


Since $n_{\varepsilon}^{\prime \prime} \geq 0$ the adjoint equation admits a unique solution. Taking the inner product of (4.8) with $p$ and of (4.10) with $D_{\vartheta} x\left(\vartheta_{\varepsilon}\right)$ we obtain

$D_{\vartheta} \mathcal{E}\left(x_{\varepsilon}\left(\vartheta_{\varepsilon}\right)\right)\left(\vartheta-\vartheta_{\varepsilon}\right)=2\left\langle p,\left[K_{k}^{T} N_{\varepsilon}^{\prime}\left(K_{k} x_{\varepsilon}\left(\vartheta_{\varepsilon}\right)\right)\right]\left(\vartheta-\vartheta_{\varepsilon}\right)\right\rangle \geq 0$ for all $\vartheta \geq 0$

or equivalently

$$
\left\langle N_{\varepsilon}^{\prime}\left(K_{k} x_{\varepsilon}\left(\vartheta_{\varepsilon}\right)\right), K_{k} p\right\rangle\left(\vartheta_{k}-\vartheta_{\varepsilon, k}\right) \geq 0 \text { for all } \vartheta_{k}>0, k=1, \ldots q .
$$

Summarizing, the necessary optimality condition for the regularized problem is given by

$$
\left\{\begin{array}{l}
x_{\varepsilon}+\sum_{k=1}^{q} \vartheta_{\varepsilon, k} K_{k}^{T} N_{\varepsilon}^{\prime}\left(K_{k} x_{\varepsilon}\right)=f \quad \text { (primal equation) } \\
p_{\varepsilon}+\sum_{k=1}^{q} \vartheta_{\varepsilon, k} K_{k}^{T} N_{\varepsilon}^{\prime \prime}\left(K_{k} x_{\varepsilon}\right) K_{k} p_{\varepsilon}=-\left(x_{\varepsilon}-g\right) \quad \text { (adjoint equation) } \\
\left\langle N_{\varepsilon}^{\prime}\left(K_{k} x_{\varepsilon}\right), K_{k} p_{\varepsilon}\right\rangle\left(\vartheta_{k}-\vartheta_{\varepsilon, k}^{*}\right) \geq 0, \text { for all } \vartheta_{k} \geq 0, k=1, \ldots, q \quad \text { (optimality) } .
\end{array}\right.
$$

The last expression in (4.13) can equally well be expressed as $N_{\varepsilon}^{\prime}\left(K_{k} x\right)^{T} K_{k} p \in$ $-\partial I_{\mathbb{R}^{+}}\left(\vartheta_{k}^{*}\right)$, where $I_{\mathbb{R}^{+}}$is the indicator function of $\mathbb{R}^{+}$and $\partial I_{\mathbb{R}^{+}}\left(\vartheta_{k}^{*}\right)$ denotes the subdifferential evaluated at $\vartheta_{k}^{*}, k=1, \ldots, r$. To obtain an optimality system for the original problem (4.1) we shall pass to the limit $\varepsilon \rightarrow 0^{+}$in (4.13). A similar procedure was used in [9] in the context of optimal control of a Bingham fluid; in this case the minimization variable appears as affine, rather than as multiplicative term like in our case and a different type of regularization was used. Alternatively a first order condition can be obtained by using the Mordukovich calculus compare [23] for mathematical programming problems with equilibrium constraints.

Theorem 4.3. Let $\vartheta^{*} \geq 0$ denote a solution to (4.1) with associated state $x^{*}=x\left(\vartheta^{*}\right)$. Then there exists an adjoint state $p \in \mathbb{R}^{n}$ and multipliers $\lambda_{k} \in$ 
$\mathbb{R}^{m}, k=1, \ldots, q$ and $\xi \in \mathbb{R}^{n}$ satisfying the following optimality system

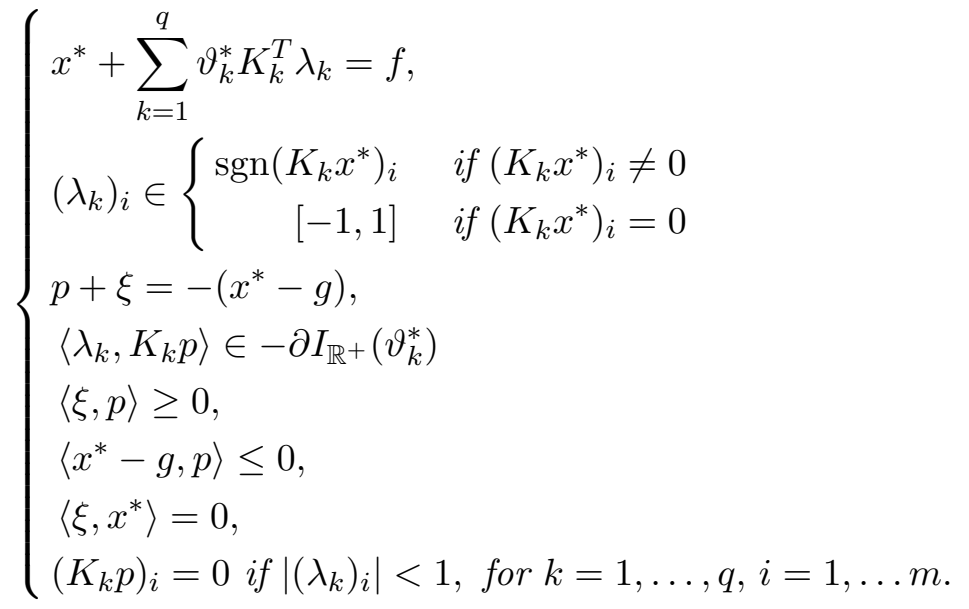

Proof. The proof of theorem 4.3 is given in the appendix.

Remark 4.4. Before closing this subsection we comment on the chosen regularization $n_{\varepsilon}$ of the norm function in (4.5), by comparing to other choices that were made in related cases. The optimality condition for the lower level problem in (4.4) with $q=1$ is given by

$$
x+\vartheta K^{T} \lambda=f
$$

where $\lambda_{i} \in \partial\left(\left|(K x)_{i}\right|\right)$, which can also be expressed as

$$
\left\{\begin{array}{l}
x+\vartheta K^{T} \lambda=f \\
|K x| \otimes \lambda=K x,|\lambda|_{\infty} \leq 1,
\end{array}\right.
$$

where $a \otimes b=\left(a_{1} b_{1}, \ldots, a_{n} b_{n}\right)$. The same system is obtained by Fenchel dualization of the lower level problem in (4.4) with $\lambda$ chosen as the dual variable. A regularization of this primal-dual formulation is obtained by replacing coordinate-wise the norm operation $|t|$ in (4.15) by $\tilde{n}_{\varepsilon}(t)=\sqrt[2]{t^{2}+\varepsilon}$. Such an approach was used for TV-regularized problems in [5], and it is also related to taught string algorithm as pointed out in [13]. Alternatively a localized regularization can be chosen by setting

$$
\hat{n}_{\varepsilon}(t)= \begin{cases}\frac{1}{2 \varepsilon} t^{2}+\frac{\varepsilon}{2} & \text { if }|t|<\varepsilon \\ |t| & \text { else },\end{cases}
$$

as used in $[9,14]$, for example. Let us compute to which kind of regularized primal formulation, the primal-dual formulation (4.15) regularized by 
(4.16) would lead, i.e. we replace the generalized derivative $\lambda \in \partial(|K x|)$ by $\frac{K x}{\hat{n}_{\varepsilon}(K x)}$, coordinate-wise, and compute the antiderivatives to obtain a new regularization $\tilde{n}_{\varepsilon}(|K x|)$. Carrying this out coordinate-wise we obtain

$$
\tilde{n}_{\varepsilon}(t)= \begin{cases}\varepsilon\left[\log \left(\frac{\varepsilon}{2}+\frac{t^{2}}{2 \varepsilon}\right)-\log \left(\frac{\varepsilon}{2}\right)\right] & \text { if }|t|<\varepsilon \\ (|t|+\varepsilon(\log (2)-1)) & \text { else } .\end{cases}
$$

This regularization of $n(t)$ is again $C^{2}$-regular with monotone derivative $\tilde{n}_{\varepsilon}^{\prime}$, which is essential for the solvability of the necessary condition associated to the lower-level problem. Differently from $(4.4), \tilde{n}_{\varepsilon}(t)$ acts globally and the expressions for the derivations are rational functions rather than polynomials. Thus we prefer (4.4) over (4.17).

\subsection{Necessary second order optimality condition}

Here we derive a second order necessary condition for local solutions of (4.4). Beyond the intrinsic relevance for describing the structure of the second order necessary condition its discussion is motivated by the fact that we introduce a second order sufficient condition in the following subsection in order to analyse a semi-smooth Newton method for solving (4.13). Of course, it is desirable that the gap between necessary and sufficient optimality condition is small. We henceforth drop the dependence of $(\vartheta, x, p)$, solution to (4.13) on $\varepsilon>0$.

In principle, the derivation of the second order conditions is quite standard, see e.g. [21], Section 10.5. Our situation, however, it is complicated due to the lack of second order smoothness of the equality constraint in (4.4). Second order conditions for general semi-smooth optimization problems were investigated for instance in [6]. Our situation here is somewhat different, however. First, only the constraints lack sufficient regularity, while the objective functional is regular, and secondly the null-space representation of the linearized equality has a special structure since the variables $x \in \mathbb{R}^{n}$ can be represented in terms of $\vartheta \in \mathbb{R}^{n}$. It is therefore appropriate to give an independent derivation.

Let $\bar{\vartheta}$ denote a local solution to (4.4) with associated state $\bar{x}=x(\bar{\vartheta})$, (i.e. the dependence of the solution on $\varepsilon>0$ is dropped here). We denote the set of strongly active indices by

$$
\overline{\mathcal{A}}_{S}=\left\{k:\left\langle N_{\varepsilon}^{\prime}\left(K_{k} \bar{x}\right), K_{k} p\right\rangle>0\right\} .
$$


On this set $\bar{\vartheta}_{k}=0$ is determined by the necessary conditions. The critical cone for the necessary second order condition is defined by

$$
\overline{\mathcal{C}}=\left\{\vartheta \in \mathbb{R}^{q}: \vartheta_{k}=0 \text { for } k \in \overline{\mathcal{A}}_{S}, \vartheta_{k} \geq 0 \text { if } \bar{\vartheta}_{k}=0\right\} .
$$

For any $\hat{\vartheta} \in \overline{\mathcal{C}}$ we have $\bar{\vartheta}+t \hat{\vartheta} \geq 0$ for all $t \geq 0$ sufficiently small. For convenience we also recall the primal equation

$$
x+\sum_{k=1}^{q} \vartheta_{k} K_{k}^{T} N_{\varepsilon}^{\prime}\left(K_{k} x\right)=f
$$

The directional derivative of $x$ with respect to $\vartheta$ at $\bar{\vartheta}$ in direction $\hat{\vartheta}$ is denoted by $\dot{x} \in \mathbb{R}^{n}$. It satisfies

$$
L_{1} \dot{x}+L_{2} \hat{\vartheta}=0
$$

Here $L_{1} \in \mathbb{R}^{n \times n}$ and $L_{2} \in R^{n \times q}$ are given by

$$
L_{1}=I+\sum_{k=1}^{q} \bar{\vartheta}_{k} K_{k}^{T} N_{\varepsilon}^{\prime \prime}\left(K_{k} \bar{x}\right) K_{k}, \quad L_{2}=\left(K_{q}^{T} N^{\prime}\left(K_{q} \bar{x}\right), \ldots, K_{q}^{T} N^{\prime}\left(K_{q} \bar{x}\right)\right) .
$$

We shall need the third derivatives of $t \rightarrow n_{\varepsilon}\left(\left(K_{k} x(\bar{\vartheta}+t \hat{\vartheta})\right)_{i}\right)$ at $t=$ 0 , which requires attention in case $\left|\left(K_{k} x(\bar{\vartheta})\right)_{i}\right|=\varepsilon$. If $\left(K_{k} x\left(\vartheta^{*}\right)\right)_{i}=\varepsilon$ and $\left.\frac{d}{d t}\left(\left(K_{k} x(\bar{\vartheta}+t \hat{\vartheta})\right)_{i}\right)\right|_{t=0}=\left(K_{k} \dot{x}\right)_{i}>0$, then by the formulas above (4.7) we have that the third order directional derivative is 0 , if on the other hand $\left(K_{k} \dot{x}\right)_{i}<0$ then the third order right directional derivative is $n_{\varepsilon}^{\prime \prime \prime}\left(\left(K_{k} x(\bar{\vartheta})\right)_{i}\right)=-\frac{3}{\varepsilon^{3}}\left(K_{k} x(\bar{\vartheta})\right)_{i}$. Finally, if $\left(K_{k} x(\dot{\vartheta})_{i}=0\right.$, then the third right directional derivative is multivalued with values in $\left\{0,-\frac{3}{\varepsilon^{3}},\left(K_{k} x(\bar{\vartheta})\right)_{i}\right\}$. Summarizing, if $n_{\varepsilon}\left(\left(K_{k} x(\bar{\vartheta})\right)_{i}\right)=\varepsilon$ then we denote the third order directional derivative of $\left.t \rightarrow n_{\varepsilon}\left(\left(K_{k} x(\bar{\vartheta}+t \hat{\vartheta})\right)_{i}\right)\right|_{t=0}$ by $n_{\varepsilon, \hat{\vartheta}}^{\prime \prime \prime}\left(K_{k} x(\bar{\vartheta})\right)_{i}$ and it is given by

$$
n_{\varepsilon, \hat{\vartheta}}^{\prime \prime \prime}\left(\left(K_{k} x(\bar{\vartheta})\right)_{i}\right) \in\left\{\begin{array}{r}
0 \text { if }\left(K_{k} \dot{x}\right)_{i}>0,\left(K_{k} x(\bar{\vartheta})\right)_{i}=\varepsilon \\
-\frac{3}{\varepsilon^{3}}\left(K_{k} x(\bar{\vartheta})\right)_{i} \text { if }\left(K_{k} \dot{x}\right)_{i}<0,\left(K_{k} x(\bar{\vartheta})\right)_{i}=\varepsilon \\
\left\{0,-\frac{3}{\varepsilon^{3}}\left(K_{k} x(\bar{\vartheta})\right)_{i}\right\} \text { if }\left(K_{k} \dot{x}\right)_{i}=0,\left(K_{k} x(\bar{\vartheta})\right)_{i}=\varepsilon .
\end{array}\right.
$$

We shall see that the expression $n_{\varepsilon, \hat{\vartheta}}^{\prime \prime \prime}\left(K_{k} x(\bar{\vartheta})\right)_{i}$ always appears as factor with $\left(K_{k} \dot{x}(\bar{\vartheta})\right)_{i}$, and we note that the expression $n_{\varepsilon, \hat{\vartheta}}^{\prime \prime \prime}\left(\left(K_{k} x(\bar{\vartheta})\right)_{i}\right)\left(K_{k} \dot{x}(\bar{\vartheta})\right)_{i}$ is 
single valued. The expressions for the case $\left(K_{k} x(\bar{\vartheta})\right)_{i}=-\varepsilon$ can be derived in a completely analogous manner. If $\left|\left(K_{k} x(\bar{\vartheta})\right)_{i}\right| \neq \varepsilon$, then the third derivative of $\left.t \rightarrow n_{\varepsilon}\left(K_{k} x(\bar{\vartheta}+t \hat{\vartheta})\right)_{i}\right|_{t=0}$ is clearly well defined. It is again denoted by $n_{\varepsilon, \hat{\vartheta}}^{\prime \prime \prime}\left(\left(K_{k} x(\bar{\vartheta})\right)_{i}\right)$. The expression for the third directional right derivative of $t \rightarrow N_{\varepsilon, \hat{\vartheta}}\left(K_{k} x(\bar{\vartheta})\right)$ is obtained from

$$
N_{\varepsilon, \hat{\vartheta}}^{\prime \prime \prime}\left(K_{k} x(\bar{\vartheta})\right)=\operatorname{diag}\left(n_{\varepsilon, \hat{\vartheta}}^{\prime \prime \prime}\left(\left(K_{k} x(\bar{\vartheta})\right)_{1}\right), \ldots, n^{\prime \prime \prime}\left(\left(K_{k} x(\bar{\vartheta})\right)_{m}\right)\right) .
$$

Associated to the local solution $(\bar{x}, \bar{\vartheta})$ we recall the adjoint equation, which we now express as

$$
L_{1} p=-(\bar{x}-g)
$$

Finally we introduce the Lagrangian associated to (4.1)

$$
\hat{\mathcal{L}}(x, \vartheta, p)=\frac{1}{2}\|x-g\|_{2}^{2}+\left\langle p, x+\sum_{k=1}^{q} \vartheta_{k} K_{k}^{T} N_{\varepsilon}^{\prime}\left(K_{k} x\right)-f\right\rangle .
$$

We are now prepared to establish a second necessary condition for (4.1) at $(\bar{x}, \bar{\vartheta})$.

Theorem 4.5. (Second order necessary condition) With the notation for $N_{\varepsilon, \hat{\vartheta}}^{\prime \prime \prime}$ introduced above we have

$$
0 \leq\left(\dot{x}^{T}, \hat{\vartheta}^{T}\right)\left(\begin{array}{cc}
I+\sum_{i=1}^{q} \bar{\vartheta}_{i} K_{i}^{T} N_{\varepsilon, \hat{\vartheta}}^{\prime \prime \prime}\left(K_{i} \bar{x}\right) \operatorname{diag}(K p) K_{i} & R \\
R^{T} & 0
\end{array}\right)\left(\begin{array}{c}
\dot{x} \\
\hat{\vartheta}
\end{array}\right)
$$

for each $\bar{\vartheta} \in \overline{\mathcal{C}}$. Here $R \in \mathbb{R}^{n \times q}$ is given by

$$
R=\left(K_{1}^{T} N_{\varepsilon}^{\prime \prime}\left(K_{1} \bar{x}\right) K_{1} p, \ldots, K_{q}^{T} N_{\varepsilon}^{\prime \prime}\left(K_{q} \bar{x}\right) K_{q} p\right)
$$

and $\dot{x}$ satisfies (4.19).

Proof. Let $\hat{\vartheta} \in \overline{\mathcal{C}}$, set $\vartheta(t)=\bar{\vartheta}+t \hat{\vartheta}$, and let $x=x(t)$ denote the solution to

$$
x+\sum_{k=1}^{q}\left(\bar{\vartheta}_{k}+t \hat{\vartheta}_{k}\right) K_{k}^{T} N_{\varepsilon}^{\prime}\left(K_{k} x\right)=f,
$$

where $t>0$, and $\dot{x}$ the solution to (4.19). In the following computation it is assumed that $t$ is sufficiently small so that $\bar{\vartheta}+t \hat{\vartheta} \geq 0$ and such that $\mathcal{E}(\vartheta(t)) \geq \mathcal{E}(\bar{\vartheta})$. Consequently we have

$$
0 \leq \hat{\mathcal{L}}(x(t), \vartheta(t), p)-\hat{\mathcal{L}}(\bar{x}, \bar{\vartheta}, p) .
$$


and moreover

$$
\nabla_{x} \hat{\mathcal{L}}(\bar{x}, \bar{\vartheta}, p)=0
$$

Therefore we find, using $|a|^{2}-|b|^{2}-2\langle a-b, b\rangle=|a-b|^{2}$, and $\hat{\vartheta} \in \overline{\mathcal{C}}$ in the first equality below, that

$$
\begin{aligned}
& 0 \leq \hat{\mathcal{L}}(x(t), \vartheta(t), p)-\hat{\mathcal{L}}(\bar{x}, \bar{\vartheta}, p)-\left\langle\nabla_{x} \hat{\mathcal{L}}(\bar{x}, \bar{\vartheta}, p), x(t)-\bar{x}\right\rangle \\
& =\frac{1}{2}\|x(t)-g\|_{2}^{2}-\frac{1}{2}\|\bar{x}-g\|_{2}^{2}-\langle x(t)-\bar{x}, \bar{x}-g\rangle \\
& +\left\langle p, \sum_{k=1}^{q}\left(\bar{\vartheta}_{k}+t \hat{\vartheta}_{k}\right) K_{k}^{T} N_{\varepsilon}^{\prime}\left(K_{k} x(t)\right)-\sum_{k=1}^{q} \bar{\vartheta}_{k} K_{k}^{T}\left(N_{\varepsilon}^{\prime}\left(K_{k} \bar{x}\right)\right)\right. \\
& \left.-\sum_{k=1}^{q} t \hat{\vartheta}_{k} K_{k}^{T} N_{\varepsilon}^{\prime}\left(K_{k} \bar{x}\right)-\sum_{k=1}^{q} \bar{\vartheta}_{k} K_{k}^{T} N^{\prime \prime}\left(K_{k} \bar{x}\right) K_{k}(x(t)-\bar{x})\right\rangle \\
& =\frac{1}{2}\|x(t)-\bar{x}\|^{2}+\left\langle p, \sum_{k=1}^{q} \bar{\vartheta}_{k} K_{k}^{T}\left(N_{\varepsilon}^{\prime}\left(K_{k} x(t)\right)-N_{\varepsilon}^{\prime}\left(K_{k} \bar{x}\right)-N_{\varepsilon}^{\prime \prime}(K \bar{x}) K_{k}(x(t)-\bar{x})\right)\right\rangle \\
& +\left\langle p, \sum_{k=1}^{q} t \hat{\vartheta}_{k} K_{k}^{T}\left(N_{\varepsilon}^{\prime}\left(K_{k} x(t)\right)-N_{\varepsilon}^{\prime}\left(K_{k} \bar{x}\right)\right)\right\rangle .
\end{aligned}
$$

By the discussion preceeding the statement of the theorem we obtain that

$$
\begin{aligned}
\lim _{t \rightarrow 0^{+}} \frac{1}{t^{2}}\left(N_{\varepsilon}^{\prime}\left(K_{k} x(t)\right)-N_{\varepsilon}^{\prime}\left(K_{k} \bar{x}\right)-N_{\varepsilon}^{\prime \prime}\left(K_{k} \bar{x}\right) K_{k}(x(t)-x)\right) \\
=\lim _{t \rightarrow 0^{+}} \frac{1}{t^{2}}\left(N_{\varepsilon}^{\prime}\left(K_{k} x(t)\right)-N_{\varepsilon}^{\prime}\left(K_{k} \bar{x}\right)-N_{\varepsilon}^{\prime \prime}\left(K_{k} \bar{x}\right) K_{k}(x(t)-\bar{x})\right. \\
\quad-\frac{1}{2} N_{\varepsilon}^{\prime \prime \prime}\left(K_{k} \bar{x}\right) \operatorname{diag}\left(K_{k}(x(t)-\bar{x})\right) K_{k}(x(t)-\bar{x}) \\
\left.\quad+\frac{1}{2} N_{\varepsilon, \hat{\vartheta}}^{\prime \prime \prime}\left(K_{k} \bar{x}\right) \operatorname{diag}\left(K_{k}(x(t)-\bar{x})\right) K_{k}(x(t)-\bar{x})\right) \\
=\frac{1}{2} N_{\varepsilon, \hat{\vartheta}}^{\prime \prime \prime}\left(K_{k} \bar{x}\right) \operatorname{diag}\left(K_{k} \dot{x}\right) K_{k} \dot{x}
\end{aligned}
$$

As a consequence we have

$$
\begin{aligned}
0 \leq & \frac{1}{2}\|\dot{x}\|^{2}+\frac{1}{2}\left\langle p, \sum_{k=1}^{q} \bar{\vartheta}_{k} K_{k}^{T} N_{\varepsilon, \hat{\vartheta}}^{\prime \prime \prime}\left(K_{k} \bar{x}\right) \operatorname{diag}\left(K_{k} \dot{x}\right) K_{k} \dot{x}\right\rangle+\left\langle p, \sum_{k=1}^{q} \hat{\vartheta}_{k} K_{k}^{T} N_{\varepsilon}^{\prime \prime}\left(K_{k} \bar{x}\right) K_{k} \dot{x}\right\rangle \\
= & \frac{1}{2}\|\dot{x}\|^{2}+\frac{1}{2} \sum_{k=1}^{q} \bar{\vartheta}_{k}\left\langle\operatorname{diag}\left(K_{k} p\right) N_{\varepsilon, \hat{\vartheta}}^{\prime \prime \prime}\left(K_{k} \bar{x}\right)\left(K_{k} \dot{x}\right), K_{k} \dot{x}\right\rangle \\
& +\sum_{k=1}^{q} \bar{\vartheta}_{k}\left\langle K_{k}^{T} N_{\varepsilon}^{\prime \prime}\left(K_{k} \bar{x}\right) K_{k} p, \bar{x}\right\rangle,
\end{aligned}
$$


which can be expressed as

$$
0 \leq\left(\dot{x}^{T}, \hat{\vartheta}^{T}\right)\left(\begin{array}{cc}
I+\sum_{k=1}^{q} \bar{\vartheta}_{k} K_{k}^{T} N_{\varepsilon, \hat{\vartheta}}^{\prime \prime \prime}\left(K_{k} \bar{x}\right) \operatorname{diag}\left(K_{k} p\right) K_{k} & R \\
R^{T} & 0
\end{array}\right)\left(\begin{array}{l}
\dot{x} \\
\hat{\vartheta}
\end{array}\right)
$$

as desired.

From the discussion before Theorem 3.1 we recall that the coordinates of $N_{\varepsilon, \hat{\vartheta}}^{\prime \prime \prime}\left(K_{i} \hat{x}\right) K_{i} \dot{x}$ and hence of $N_{\varepsilon, \hat{\vartheta}}^{\prime \prime \prime}\left(K_{i} \hat{x}\right) \operatorname{diag}(K p) K_{i} \dot{x}$ are single valued.

\subsection{Semi-smooth Newton algorithms}

In this subsection a semi-smooth Newton method for solving the necessary optimality system (4.13) for the regularized problem (4.4) is developed and analyzed. Convergence of the regularized problem to the original one was already studied in Theorem 4.3. We utilize that the optimality condition in (4.11) can equivalently be expressed by means of the complementarity formulation

$$
\begin{array}{r}
\left(\left\langle N_{\varepsilon}^{\prime}\left(K_{k} x\right), K_{k} p\right\rangle\right)_{q \times 1}-\mu=0 \\
\mu-\max (0, \mu-c \vartheta)=0,
\end{array}
$$

where

$$
\left(\left\langle N_{\varepsilon}^{\prime}\left(K_{k} x\right), K_{k} p\right\rangle\right)_{q \times 1}=\left(\left\langle N_{\varepsilon}^{\prime}\left(K_{1} x\right), K_{1} p\right\rangle, \ldots,\left\langle N_{\varepsilon}^{\prime}\left(K_{r} x\right), K_{r} p\right\rangle\right),
$$

$c$ is any positive scalar and max operates coordinate-wise.

System (4.11) can therefore be expressed equivalently as

$$
G(x, \vartheta, p, \mu)=0
$$

where

$$
G(x, \vartheta, p, \mu)=\left(\begin{array}{c}
p+\sum_{k=1}^{q} \vartheta_{k} K_{k}^{T} N_{\varepsilon}^{\prime \prime}\left(K_{k} x\right) K_{k} p+x-g \\
\left(\left\langle N_{\varepsilon}^{\prime}\left(K_{k} x\right), K_{k} p\right\rangle\right)_{q \times 1}-\mu \\
\sum_{k=1}^{q} \vartheta_{k} K_{k}^{T} N_{\varepsilon}^{\prime}\left(K_{k} x\right)+x-f \\
\mu-\max (0, \mu-c \vartheta)
\end{array}\right) .
$$

The reason for exchanging the order of the equations is to create symmetries in the generalized Jacobian $J(x, \vartheta, p, \mu)$ of $G(x, \vartheta, p, \mu)$ to be specified below. 
In what follows we specify the value of $n^{\prime \prime \prime}(t)$ at $t= \pm \varepsilon$ to be 0 . We could equally well take $\mp \frac{3}{\varepsilon^{2}}$. For $(x, \vartheta, p, \mu) \in \mathbb{R}^{n} \times \mathbb{R}^{q} \times \mathbb{R}^{n} \times \mathbb{R}^{q}$ we define

$L_{1}(x, \vartheta)=I+\sum_{k=1}^{q} \vartheta_{k} K_{k}^{T} N_{\varepsilon}^{\prime \prime}\left(K_{k} x\right) K_{k}, L_{2}(x)=\left(K_{1}^{T} N_{\varepsilon}^{\prime}\left(K_{1} x\right), \ldots, K_{q}^{T} N_{\varepsilon}^{\prime}\left(K_{q} x\right)\right)$,

$R(x)=\left(K_{1}^{T} N_{\varepsilon}^{\prime \prime}\left(K_{k} x\right) K_{1} p, \ldots, K_{q}^{T} N_{\varepsilon}^{\prime \prime}\left(K_{q} x\right) K_{r} p\right) \in \mathbb{R}^{n \times q}$,

$\operatorname{Max}^{\prime}(0, \mu)=\operatorname{diag}\left(\max ^{\prime}\left(0, \mu_{1}\right), \ldots, \max ^{\prime}\left(0, \mu_{q}\right)\right)$,

where

$$
\max ^{\prime}\left(0, \mu_{k}\right)=\left\{\begin{array}{l}
1 \text { if } \mu_{k}>0 \\
0 \text { if } \mu_{k} \leq 0
\end{array}\right.
$$

We note that there exists a neighborhood $U$ We find that the generalized Jacobian of $G$ is given by

$$
J(x, \vartheta, p, \mu)=\left(\begin{array}{cccc}
Q(x, \vartheta, p) & R(x) & L_{1}(x, \vartheta) & 0 \\
R(x)^{T} & 0 & L_{2}^{T}(x) & -I \\
L_{1}(x, \vartheta) & L_{2}(x) & 0 & 0 \\
0 & c \operatorname{Max}^{\prime}(0, \mu-c \vartheta) & 0 & I-\operatorname{Max}^{\prime}(0, \mu-c \vartheta)
\end{array}\right) \text {, }
$$

where

$$
Q(x, \vartheta, p)=I+\sum_{k=1}^{q} \vartheta_{k} K_{k}^{T} N_{\varepsilon}^{\prime \prime \prime}\left(K_{k} x\right) \operatorname{diag}\left(K_{k} p\right) K_{k} .
$$

A positive definiteness assumption of the upper $2 \times 2$ block of $J(x, \vartheta, p, \mu)$ will be required. Let $(\bar{x}, \bar{\vartheta}, \bar{p}, \bar{\mu})$ denote a solution to $G(x, \vartheta, p, \mu)=0$. Further let $U=U(\bar{x}, \bar{\vartheta}, \bar{p}, \bar{\mu})$ denote an open neighborhood of $(\bar{x}, \bar{\vartheta}, \bar{p}, \bar{\mu})$ and set

$$
\mathcal{A}(\vartheta, \mu)=\left\{k: \mu_{k}-c \vartheta_{k}>0\right\},
$$

and

$$
\mathcal{C}=\left\{\delta \vartheta \in \mathbb{R}^{q}: \delta \vartheta_{k}=0 \text { if } k \in \mathcal{A}(\vartheta, \mu)\right\} .
$$

Note that at the solution we have $\bar{\vartheta}_{k} \bar{\mu}_{k}=0$, and $\bar{\mu}_{k} \geq 0, \bar{\vartheta}_{k} \geq 0$, and hence $\mathcal{A}(\bar{\vartheta}, \bar{\mu})=\left\{k: \bar{\mu}_{k}>0\right\}$ coincides with the strongly active set of Section 3.3. We shall utilize the following assumption:

There exists a bounded neighborhood $U=U(\bar{x}, \bar{\vartheta}, \bar{p}, \bar{\mu})$ such that for all $(x, \vartheta, p, \mu) \in U$

$$
\left(\delta x^{T}, \delta \vartheta^{T}\right)\left(\begin{array}{cc}
Q(x, \vartheta, p) & R(x) \\
R(x) & 0
\end{array}\right)\left(\begin{array}{l}
\delta x \\
\delta \vartheta
\end{array}\right)>0
$$

for all $\vartheta \in \mathcal{C}$ and $L_{1}(x, \vartheta) \delta x+L_{2}(x) \delta \vartheta=0$. 
Note that $\mu$ does not appear in the positive definite condition, but boundedness of the $\mu$-coordinates in $U$ will be used below.

Comparing (H1) to the second order necessary condition of Theorem 4.5 we note that (H1) requires positive definiteness in a neighborhood of $(\bar{x}, \bar{\vartheta}, \bar{p})$, that perturbations of the active set need to be admitted and that the values of $n_{\varepsilon}^{\prime \prime \prime}$ at $t= \pm \varepsilon$ are fixed, whereas in Theorem 4.5 they appear as directional derivatives. For the purpose of this subsection the choice of $\max ^{\prime}\left(0, \mu_{k}\right)$ could be 0 at $\mu_{k}=0$. This would change $\mathcal{A}(\vartheta, \mu)=\left\{k: \mu_{k}-c \vartheta_{k} \geq 0\right\}$, but the following convergence result would remain unchanged.

By $(\mathrm{H} 1)$ and the fact that $t \rightarrow n_{\varepsilon}^{\prime \prime \prime}(t)$ has only finitely many discontinuities, there exists $\kappa>0$ such that

$$
\left(\delta x^{T}, \delta \vartheta^{T}\right)\left(\begin{array}{cc}
Q(x, \vartheta, p) & R(x) \\
R^{T}(x) & 0
\end{array}\right)\left(\begin{array}{l}
\delta x \\
\delta \vartheta
\end{array}\right) \geq \kappa\left\|\left(\begin{array}{l}
\delta x \\
\delta \vartheta
\end{array}\right)\right\|_{2}^{2},
$$

for all $\vartheta \in \mathcal{C}, L_{1}(x, \vartheta) \delta x+L_{2}(x) \delta \vartheta=0$, and $(x, \vartheta, p, \mu) \in U$.

Proposition 4.6. If $(\mathrm{H} 1)$ holds, then $J(x, \vartheta, p, \mu)$ is regular for each $(x, \vartheta, p, \mu) \in$ $U$ and the inverses are uniformly bounded.

Proof. Let $(x, \vartheta, p, \mu) \in U$, set $\mathcal{A}=\mathcal{A}(\vartheta, \mu)$ as definded above, and let $\mathcal{I}=$ $\{1, \ldots r\} \backslash \mathcal{A}$. We show that $J(x, \vartheta, p, \mu)$ is injective. Let $(\delta x, \delta \vartheta, \delta p, \delta \mu) \in$ $\mathbb{R}^{n} \times \mathbb{R}^{q} \times \mathbb{R}^{n} \times \mathbb{R}^{q}$ and assume that

$$
J(x, \vartheta, p, \mu)\left(\begin{array}{l}
\delta x \\
\delta \vartheta \\
\delta p \\
\delta \mu
\end{array}\right)=0
$$

We partition $\delta \vartheta$ into coordinates associated to inactive $\delta \vartheta_{\mathcal{I}}$ and active $\delta \vartheta_{\mathcal{A}}$ coordinates, and similarily for $\delta \mu$. The columns of $R(x)$ corresponding to inactive coordinates are denoted by $R(x)_{\mathcal{I}}$ and analogously for $L_{2}(x)_{\mathcal{I}}$. Thus $R(x)_{\mathcal{I}}$ is of dimension $n \times \#(\mathcal{I})$, where $\#(\mathcal{I})$ denotes the cardinality of $\mathcal{I}$. From the last equation in (4.26) we have

$$
\delta \vartheta_{\mathcal{A}}=0 \text { and } \delta \mu_{\mathcal{I}}=0 .
$$

From the third equation in (4.26) we have

$$
L_{1}(x, \vartheta) \delta x+L_{2}(x) \delta \vartheta=L_{1}(x, \vartheta) \delta x+L_{2}(x)_{\mathcal{I}}(\delta \vartheta)_{\mathcal{I}}=0 .
$$

Now from equations one and two of (4.26)

$$
\begin{aligned}
Q(x, \vartheta, p) \delta x+R(x)_{\mathcal{I}} \delta \vartheta_{\mathcal{I}} & =-L_{1}^{T}(x, \vartheta) p \\
\left(R(x)_{\mathcal{I}}\right)^{T} \delta x & =-\left(L_{2}(x)_{\mathcal{I}}\right)^{T} p
\end{aligned}
$$


where we use that $(\delta \mu)_{\mathcal{I}}=0$. Since by $(\mathrm{H} 1)$ the matrix $\left(\begin{array}{cc}Q & R_{\mathcal{I}} \\ R_{\mathcal{I}}^{T} & 0\end{array}\right)$ is positive definite on $\operatorname{ker}\left(L_{1}(x, \vartheta), L_{2}(x)_{\mathcal{I}}\right)$ and the right hand side is in its orthogonal complement, we find $\delta x=0$ and $\delta \vartheta_{\mathcal{I}}=0$. From the first equation in (4.26) we deduce that $\delta p=0$. The third equation, evaluated for the $\mathcal{A}$-coordinates implies that $\delta \mu_{\mathcal{A}}=0$, and hence $J(x, \vartheta, p, \mu)$ is a regular matrix. Since $U$ is bounded, its closure is compact. Now by a compactness argument, and the fact that $J(x, \vartheta, p, \mu)$ has at most finitely many discontinuities in $x$, uniform boundedness of the inverses follows.

A semi-smooth Newton algorithm for solving $G(x, \vartheta, p, \mu)=0$ can now be specified.

\begin{tabular}{l}
\hline Algorithm 4.1 Newton Learning for $\ell_{1}\left(\mathrm{NL}-\ell_{1}\right)$ \\
\hline (i) Choose $\left(x^{0}, \vartheta^{0}, p^{0}, \mu^{0}\right) \in \mathbb{R}^{n} \times \mathbb{R}^{q} \times \mathbb{R}^{n} \times \mathbb{R}^{q}$, set $n=0$, \\
(ii) Solve $J\left(x^{n}, \vartheta^{n}, p^{n}, \mu^{n}\right)\left(\begin{array}{c}\delta x \\
\delta \vartheta \\
\delta p \\
\delta \mu\end{array}\right)=-G\left(x^{n}, \vartheta^{n}, p^{n}, \mu^{n}\right)$, \\
(iii) Update $\left(x^{n+1}, \vartheta^{n+1}, p^{n+1}, \mu^{n+1}\right)=\left(x^{n}, \vartheta^{n}, p^{n}, \mu^{n}\right)+(\delta x, \delta \vartheta, \delta p, \delta \mu)$, \\
set $n=n+1$ and goto (ii).
\end{tabular}

Theorem 4.7. Let $(\bar{x}, \bar{\vartheta}, \bar{p}, \bar{\mu})$ denote a solution of $G(x, \vartheta, p, \mu)=0$ and assume that (H1) holds. Then Algorithm 4.1 converges locally superlinearly.

Proof. Using wellknown characterizations for semi-smooth functions, see e.g. [30] pg. 27, it can be argued that $G$ is semi-smooth. Together with uniform boundedness of the generalized Jacobians $J(x, \vartheta, p, \mu)$ in a neighborhood of $(\bar{x}, \bar{\vartheta}, \bar{p}, \bar{\mu})$ the claim follows, see e.g. [30], pg. 29.

In numerical optimization Algorithm 4.1, which arises as Newton algorithm applied to the optimality condition, is frequently referred to as sequential quadratic programming (SQP-) algorithm. The algorithm can equivalently be obtained be iteratively minimizing a quadratic approximation to the cost and a linear (in $x$ and $\vartheta$ ) approximation to the constraining equation, which in our case is the necessary optimality condition to the lower level problem. Algorithm 4.1 is closely related to applying a Newton algorithm to the reduced functional $\vartheta \rightarrow \mathcal{E}(x(\vartheta))$, where $x(\vartheta)$ satisfied 
the nonlinear constraining equation. They differ by the property that the primal updates of the Newton algorithm applied to the reduced functional $\vartheta \rightarrow \mathcal{E}(x(\vartheta))$ live on the nonlinear constraining manifold, while iterates of Algorithm 4.1 are contained in the tangent space to the constraint at the current iterate. It is well-known that the former of these two algorithms can obtained from the latter by introducing feasibility steps, [16]. For the current problem this is given in Algorithm 4.2.

Algorithm 4.2 Reduced Newton Learning for $\ell_{1}\left(\mathrm{RNL}-\ell_{1}\right)$

(i) Choose $\left(\vartheta^{0}, \mu^{0}\right) \in \mathbb{R}^{q} \times \mathbb{R}^{q}$, set $n=0$,

(ii) Solve $x+\sum_{k=1}^{q} \vartheta^{n} K_{k}^{T} N_{\varepsilon}^{\prime}\left(K_{k} x\right)=f$ for $x^{n}$ (primal feasibility step),

(iii) Solve $L_{1}\left(x^{n}, \vartheta^{n}\right) p=-\left(x^{n}-g\right)$ for $p^{n}$ (dual feasibility step),

(iv) Solve $J\left(x^{n}, \vartheta^{n}, p^{n}, \mu^{n}\right)\left(\begin{array}{c}\delta x \\ \delta \vartheta \\ \delta p \\ \delta \mu\end{array}\right)=-G\left(x^{n}, \vartheta^{n}, p^{n}, \mu^{n}\right)$,

(v) Update $\left(\vartheta^{n+1}, \mu^{n+1}\right)=\left(\vartheta^{n}, \mu^{n}\right)+(\delta \vartheta, \delta \mu)$, set $n=n+1$ and goto (ii).

Due to the feasibility steps the right hand side of step (iii) in Algorithm 4.2 has the form

$$
G(x, \vartheta, p, \mu)=\left(\begin{array}{c}
0 \\
\left(\left\langle N_{\varepsilon}^{\prime}\left(K_{k} x\right), K_{k} p\right\rangle\right)_{q \times 1}-\mu \\
0 \\
\mu-\max (0, \mu-c \vartheta)
\end{array}\right) .
$$

For any solution $(\bar{x}, \bar{\vartheta}, \bar{p}, \bar{\mu})$ to $G(x, \vartheta, p, \mu)=0$ we have $\bar{\vartheta} \geq 0$. Hence $L_{1}(\bar{x}, \bar{\vartheta})$ is positive definite, and the implicit function theorem implies the existence neighborhoods $U(\bar{x}) \times U(\bar{\vartheta})$ such that for each $\vartheta \in U(\bar{\vartheta})$ there exists a solution $x=x(\vartheta) \in U(\bar{x})$ satisfying

$$
x+\sum_{k=1}^{q} \vartheta_{k} K_{k}^{T} N_{\varepsilon}^{\prime}\left(K_{k} x\right)-f=0 .
$$


Moreover $\vartheta \rightarrow x(\vartheta)$ is continuously differentiable from $U(\bar{\vartheta})$ to $U(\bar{x})$. The solution in step(ii) of Algorithm 4.2 is chosen to satisfy $x^{n} \in U(\bar{x})$. Without loss of generality we may assume that $\{(x, \vartheta):(x, \vartheta, p, \mu) \in U((\bar{x}, \bar{\vartheta}, \bar{p}, \bar{\mu}))\} \subset$ $U(\bar{x}) \times U(\bar{\vartheta})$, where $U(\bar{x}, \bar{\vartheta}, \bar{p}, \bar{\mu})$ was introduced in (H1), and that $L_{1}(x, \vartheta)$ is regular with uniformly bounded inverses for all $(x, \vartheta) \in U(\bar{x}) \times U(\bar{\vartheta})$. For nonnegative $\vartheta$ this property is obviously satisfied for all $x$.

In numerical practice we switched from Algorithm 4.2 to Algorithm ?? for small values of epsilon (e.g. $\varepsilon \leq 10^{-2}$ ). Moreover we used a reduced form of the system in step (iii) which will be detailed after addressing convergence for Algorithm 4.2.

Theorem 4.8. Let $(\bar{x}, \bar{\vartheta}, \bar{p}, \bar{\mu})$ be a solution to $G(x, \vartheta, p, \mu)=0$. If (H1) holds, then the iterates of Algorithm 4.2 converge locally superlinearly.

Proof. The proof can be given by standard arguments and hence it suffices to give the main steps.

The iteration can be characterized by

$$
z^{n} \rightarrow \hat{z}^{n+1}=z^{n}+\delta z \rightarrow z^{n+1}=\left(x^{n+1}, \vartheta^{n}+\delta \vartheta, p^{n+1}, \mu^{n}+\delta \mu\right),
$$

where $z^{n}=\left(x^{n}, \vartheta^{n}, p^{n}, \mu^{n}\right)$, and $\delta z=(\delta x, \delta \vartheta, \delta p, \delta \mu)$ is the solution to the system in step (iii) in Algorithm 4.2. The first step in (4.30) is a semi-smooth Newton step and hence

$$
\left\|z^{n}+\delta z-\bar{z}\right\|=o\left(\left\|z^{n}-\bar{z}\right\|\right),
$$

where $\bar{z}=(\bar{x}, \bar{\vartheta}, \bar{p}, \bar{\mu})$, and the norm $\|\cdot\|$ is taken in $\mathbb{R}^{n} \times \mathbb{R}^{q} \times \mathbb{R}^{n} \times \mathbb{R}^{q}$. Arguing iteratively, (4.31) together with the Lipschitz estimates below, we find that the iterates $z^{n} \in U(\bar{x}, \bar{\vartheta}, \bar{p}, \bar{\mu})$, if $\left\|\left(x^{0}, \vartheta^{0}\right)-(\bar{x}, \bar{\vartheta})\right\|$ is sufficiently small.

Since $\vartheta \rightarrow x(\vartheta)$ is $C^{1}$ on $U(\bar{\vartheta})$ there exists as constant $K_{1}$ such that $\|x(\vartheta)-\bar{x}\| \leq K_{1}\|\vartheta-\bar{\vartheta}\|$ for all $\vartheta \in U(\vartheta)$, and in particular

$$
\left\|x\left(\vartheta^{n+1}\right)-\bar{x}\right\|=\left\|x^{n+1}-\bar{x}\right\| \leq K_{1}\left\|\vartheta^{n+1}-\bar{\vartheta}\right\|=o\left(\left\|\vartheta^{n}-\bar{\vartheta}\right\|\right) .
$$

Moreover we find that

$$
L_{1}\left(x^{n+1}, \vartheta^{n+1}\right)\left(p^{n+1}-\bar{p}\right)=-\left(L_{1}\left(x^{n+1}, \vartheta^{n+1}\right)-L_{1}(\bar{x}, \bar{\vartheta})\right) \bar{p}+\bar{x}-x^{n+1},
$$

and therefore there exists a constant $K_{2}$, independent of $n$, such that

$$
\left\|p^{n+1}-\bar{p}\right\| \leq K_{2}\left\|\left(x^{n+1}, \vartheta^{n+1}\right)-(\bar{x}, \bar{\vartheta})\right\|=o\left(\left\|\vartheta^{n}-\bar{\vartheta}\right\|\right) .
$$

Combining (4.31) - (4.33) the claim follows. 
We next express step (iv) of Algorithm 4.2 in terms of the variables $(\vartheta, \mu)$. From the first and third equations of (iv) we derive

$$
\begin{aligned}
& \delta p=-L_{1}^{-1} Q \delta x+R \delta \vartheta \\
& \delta \vartheta=-L_{1}^{-1} L_{2} \delta x .
\end{aligned}
$$

Here and below we drop the dependence of $L_{1}, L_{2}, R$ and $Q$ on the current iterate $\left(x^{n}, \vartheta^{n}, p^{n}\right)$. The second equation of (iv) gives

$$
R^{T} \delta x+L_{2}^{T} \delta p-\delta \mu=-G_{3} .
$$

Introducing the symmetric matrix

$$
P\left(x^{n}, \vartheta^{n}, p^{n}\right)=L_{2}^{T} L_{1}^{-1} Q L_{1}^{-1} L_{2}-R^{T} L_{1}^{-1} L_{2}-L_{2}^{T} L_{1}^{-1} R,
$$

and $\delta x$ and $\delta \mu$ in terms of $\delta \vartheta$ in (4.34) we obtain

$$
P\left(x^{n}, \vartheta^{n}, p^{n}\right) \delta \vartheta-\delta \mu=-G_{2}\left(x^{n}, p^{n}, \mu^{n}\right) .
$$

Combined with the forth equation in (iii) we obtain the asymmetric system (4.35)

$$
\left(\begin{array}{cc}
P\left(x^{n}, \vartheta^{n}, p^{n}\right) & -I \\
c \operatorname{Max}^{\prime}\left(0, \mu^{n}-c \vartheta^{n}\right) & I-\operatorname{Max}^{\prime}\left(0, \mu^{n}-c \vartheta^{n}\right)
\end{array}\right)\left(\begin{array}{l}
\delta \vartheta \\
\delta \mu
\end{array}\right)=\left(\begin{array}{c}
-G_{2}\left(x^{n}, p^{n}, \mu^{n}\right) \\
-G_{4}\left(\vartheta^{n}, \mu^{n}\right)
\end{array}\right) .
$$

The second equality (4.35) can be expressed as $c \operatorname{Max}^{\prime}\left(0, \mu^{n}-c \vartheta^{n}\right) \delta \vartheta+\left(I-\operatorname{Max}^{\prime}\left(0, \mu^{n}-c \vartheta^{n}\right)\right) \delta \mu+\mu^{n}-\max \left(0, \mu^{n}-c \vartheta^{n}\right)=0$. This implies that

$$
\vartheta_{\mathcal{A}}^{n+1}=0 \quad \text { and } \quad \mu_{\mathcal{I}}^{n+1}=0,
$$

where $\mathcal{A}=\mathcal{A}\left(\vartheta^{n}, \mu^{n}\right)$ is defined in (4.25) with $(\vartheta, \mu)$ replaced by $\left(\vartheta^{n}, \mu^{n}\right)$ and the subscript $\mathcal{A}$ with $\vartheta_{\mathcal{A}}^{n+1}$ was defined in the proof of Proposition 4.6.

Finally we partition the coordinates into active and inactive ones, so that, after possible reordering, $x=\left(x_{\mathcal{I}}, x_{\mathcal{A}}\right)$. Accordingly $P\left(x^{n}, \vartheta^{n}, p^{n}\right)$ is split into block matrices

$$
P\left(x^{n}, \vartheta^{n}, p^{n}\right)=\left(\begin{array}{cc}
P\left(x^{n}, \vartheta^{n}, p^{n}\right)_{\mathcal{I}} & P\left(x^{n}, \vartheta^{n}, p^{n}\right)_{\mathcal{I}, \mathcal{A}} \\
P\left(x^{n}, \vartheta^{n}, p^{n}\right)_{\mathcal{A}, \mathcal{I}} & P\left(x^{n}, \vartheta^{n}, p^{n}\right)_{\mathcal{A}}
\end{array}\right) .
$$

Thus (4.35) is equivalent to solving the symmetric system

$$
P\left(x^{n}, \vartheta^{n}, p^{n}\right)_{\mathcal{I}} \delta \vartheta_{\mathcal{I}}=-\left\langle N_{\varepsilon}^{\prime}\left(K_{i} x\right), K_{i} p\right\rangle_{\mathcal{I}}+P\left(x^{n}, \vartheta^{n}, p^{n}\right)_{\mathcal{I}, \mathcal{A}} \vartheta_{\mathcal{A}}^{n},
$$

where we use that $\delta \vartheta_{\mathcal{A}}=-\vartheta_{\mathcal{A}}$, and assigning

$$
\mu_{\mathcal{A}}^{n+1}=(P \delta \vartheta)_{\mathcal{A}}+\left\langle N_{\varepsilon}^{\prime}\left(K_{i} x\right), K_{i} p\right\rangle_{\mathcal{A}},
$$

and $\vartheta_{\mathcal{A}}^{n+1}, \mu_{\mathcal{I}}^{n+1}=0$ according to $(4.36)$. 


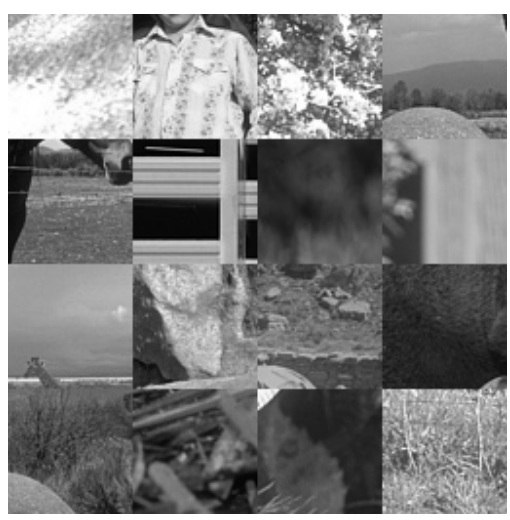

(a)

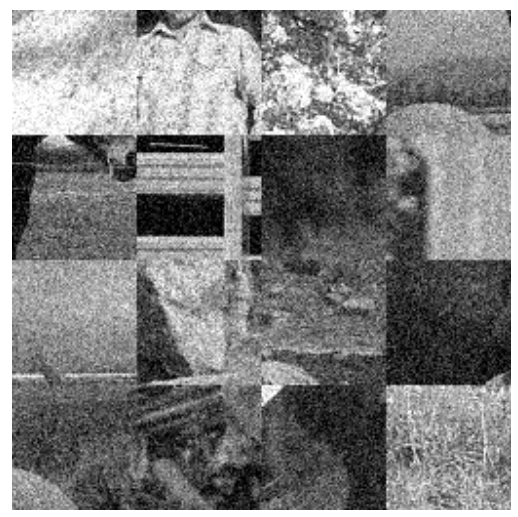

(b)

Figure 4: Subset of the ground truth data $g$ extracted from the BSDS500 database [1] and the noisy data $f$ using a noise level of $\sigma=25$.

\section{Numerical realization}

In our numerical experiments, we consider the problem of learning the optimal regularization parameters for the $\ell_{1}$ model with multiple priors from a set of training data $\left(g_{i}, f_{i}\right), i=1 \ldots N$,

$$
\left\{\begin{array}{l}
\min _{\vartheta \geq 0} \mathcal{E}(x(\vartheta))=\sum_{i=1}^{N}\left\|x_{i}(\vartheta)-g_{i}\right\|_{2}^{2} \\
\text { subject to } x_{i}(\vartheta)=\underset{x}{\operatorname{argmin}} \sum_{k=1}^{q} \vartheta_{i}\left\|K_{k} x\right\|_{1}+\frac{1}{2}\left\|x-f_{i}\right\|_{2}^{2} .
\end{array}\right.
$$

To generate the training data, we first randomly sample $N=64$ patches of size $w \times h=64 \times 64$ from the BSDS500 image segmentation database [1] and store them into vectors $g_{i} \in \mathbb{R}^{w h}$. The reason for sampling random patches in a large database is to generate samples of a large diversity by simultaneously minimizing the amount of training data. Then, we generate the noisy versions $f_{i} \in \mathbb{R}^{\text {wh }}$ by adding Gaussian noise with different standard deviations $\sigma \in\{15,25,50\}$ to $g_{i}$. Figure 4 shows an exemplary subset of the training data together with a noisy version.

In previous sections, we did not consider the case of multiple training data $\left(g_{i}, f_{i}\right)$. However, we can easily convert the learning problem for multiple training data to the form (4.1) by stacking up all $g_{i}$ and $f_{i}$ to large vectors, i.e. $\tilde{g}=\left(g_{1}, \ldots, g_{N}\right)$ and $\tilde{f}=\left(f_{1}, \ldots, f_{N}\right)$ and by defining the linear 


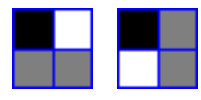

(a) $1^{s t}$
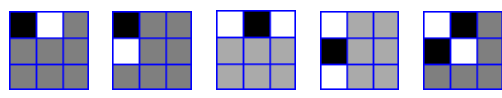

(b) $1^{\text {st }}+2^{\text {nd }}$

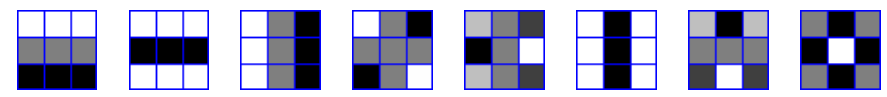

(c) $D C T 3$
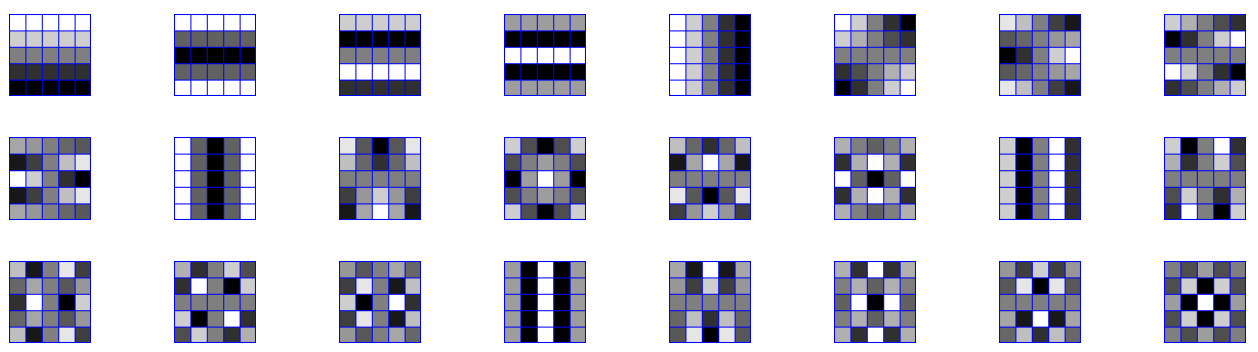

(d) $D C T 5$

Figure 5: Different sets of filters used in the experiments.

operators $\widetilde{K}_{k}$ as the $N \times N$ block-diagonal matrices

$$
\widetilde{K}_{k}=\operatorname{diag}(\underbrace{K_{k}, \ldots, K_{k}}_{N \text { times }}) \text {. }
$$

Then, we can treat the multiple training data problem as a single training data problem with a modified linear operator $\widetilde{K}$ and the analysis carried out in the previous sections can be applied.

The linear operators $K_{k} \in \mathbb{R}^{m \times n}$ we consider in our experiments are generated from local filter kernels $\kappa_{k} \in \mathbb{R}^{\mu \times \nu}$ such that the matrix-vector product $K_{k} x$ is equal to the two dimensional convolution of the two-dimensional image $x$ with the filter kernel $\kappa_{k}$, i.e.

$$
K_{k} x=x * \kappa_{k},
$$

where $*$ denotes the two-dimensional convolution operation. Note that for the matrix vector product $K_{k} x$, the image is treated as a column vector 
whereas for the two-dimensional convolution with the filter kernels $\kappa_{k}$, the image is treated as a two-dimensional array.

For the filter kernels we consider various choices, e.g. standard finite difference approximations of first- and second-order derivatives or higherorder linear operators obtained from the basis vectors of the two-dimensional discrete cosine transform (DCT). Figure 5 shows the filter kernels we used in our experiments. For the boundary conditions, we modify the linear operators in a way such that the image data is reflected at the boundaries.

\subsection{Learning}

In the following sections we show how to learn the optimal regularization parameters $\vartheta$ in the multiple prior $\ell_{1}$ model. We shall study two approaches: A first approach that reduces the $\ell_{1}$ learning problem to a sequence of reweighted $\ell_{2}$ learning problems which will be solved using Algorithm 3.1 and a second approach that directly solves the $\ell_{1}$ learning problem using the reduced Newton algorithm 4.2. We will compare the performances of both approaches and finally show preliminary extensions to solving the opti-

mal parameters of a non-convex $\ell_{\frac{1}{2}}$ model. All algorithms are implemented in Matlab and are executed on a $2.60 \mathrm{GHz}$ i5 $\mathrm{CPU}$ running a $64 \mathrm{Bit}$ Linux system.

\subsubsection{Iteratively reweighted $\ell_{2}$ learning}

Motivated by the fixed point algorithm for solving the lower-level $\ell_{1}$ problems $[32,8]$, we consider a sequence of reweighted $\ell_{2}$ problems for learning the optimal regularization parameters of the $\ell_{1}$ problem.

Let $n_{\varepsilon}$ be the $\varepsilon$ regularized Huber- $\ell_{1}$ norm

$$
n_{\varepsilon}(t)= \begin{cases}\frac{t^{2}}{2 \varepsilon}+\frac{\varepsilon}{2} & \text { if }|t| \leq \varepsilon \\ |t| & \text { else . }\end{cases}
$$

Given a point $\hat{t}$, we can bound $n_{\varepsilon}(t)$ from above via the quadratic function [3]

$$
n_{\varepsilon}(t) \leq \frac{1}{2}\left(\frac{t^{2}}{\max (\varepsilon,|\hat{t}|)}+\max (\varepsilon,|\hat{t}|)\right) .
$$

Now, assume, we are given an $\hat{x}$ which is sufficiently close to the optimal solution of the lower level problem. We can then approximate the $\ell_{1}$ bilevel learning problem as a quadratic single level problem

$$
\min _{\vartheta \geq 0} \mathcal{E}(\vartheta)=\frac{1}{2}\left\|\left(I+\sum_{k=1}^{q} \vartheta_{k} \mathcal{K}_{k}(\hat{x})\right)^{-1} f-g\right\|_{2}^{2},
$$


where

$$
\mathcal{K}_{k}(\hat{x})=K_{k}^{T} \operatorname{diag}\left(\frac{1}{\max \left(\varepsilon,\left|\left(K_{k} \hat{x}\right)_{1}\right|\right)}, \ldots, \frac{1}{\max \left(\varepsilon,\left|\left(K_{k} \hat{x}\right)_{m}\right|\right)}\right) K_{k},
$$

is the weighted linear operator. This motivates an iterative algorithm which starts with an initial estimate of $\hat{x}$ and then solves a sequence of quadratic single level problems with iteratively updated versions of $\hat{x}$. The outline of algorithm is presented in Algorithm 5.1. The most involved step in the

Algorithm 5.1 Iteratively Reweighted Learning for $\ell_{2}\left(\right.$ IRL- $\left.\ell_{2}\right)$

(i) Set $n=0, \vartheta=0, \hat{x}=f$

(ii) Compute $\mathcal{K}_{k}(\hat{x})=K_{k}^{T} \operatorname{diag}\left(\frac{1}{\max \left(\varepsilon,\left|\left(K_{k} \hat{x}\right)_{1}\right|\right)}, \ldots, \frac{1}{\max \left(\varepsilon,\left|\left(K_{k} \hat{x}\right)_{m}\right|\right)}\right) K_{k}$

(iii) Solve

$$
\vartheta^{n}=\arg \min _{\vartheta \geq 0} \mathcal{E}(\vartheta)=\frac{1}{2}\left\|\left(I+\sum_{k=1}^{q} \vartheta_{k} \mathcal{K}_{k}(\hat{x})\right)^{-1} f-g\right\|_{2}^{2}
$$

using Algorithm 3.1

(iv) Compute $\hat{x}=\left(I+\sum_{k=1}^{q} \vartheta_{k}^{n} \mathcal{K}_{k}(\hat{x})\right)^{-1} f$

(v) Set $n=n+1$, goto (ii)

algorithm is computing the solution of the weighted $\ell_{2}$ single level problem which is carried out by using the semi-smooth Newton Algorithm 3.1. In our experiments, we observe that the Hessian matrix $M$ involved in the Newton equation (3.21) can have negative eigenvalues which means that the Newton direction is not a descend direction. In view of the higher level function $\mathcal{E}(\vartheta)$ as depicted in Figure 1, this comes as no suprise given the concave behavior of $\mathcal{E}(\vartheta)$ away from zero. In this case we use a positive definite approximation of $M$ by flipping the signs of the negative eigenvalues (see [22] for more details). It is important to point out that $M$ is always positive definite when the iterate becomes sufficiently close to the optimal solution which enables the algorithms local superlinear convergence. During the iterations of Algorithm 3.1, we always perform full steps in $\mu$ and in $\vartheta_{i}, \forall i \in \mathcal{A}^{n}$ and carry out a Armijo-type linesearch in $\vartheta_{i}, \forall i \in \mathcal{I}^{n}$ using the function value of the higher level optimization problem as the merit function. We set $\varepsilon=10^{-3}$ 
in the Huber-regularized $|\cdot|$ function in (5.2). The iterations of the inner Algorithm 3.1 are stopped, when a maximum number of inner iterations maxiter $_{1}=100$ is reached or the normalized residual, i.e. the $\ell_{2}$ norm of the right hand side of (3.21) devided by its number of elements is less than a tolerance of $\operatorname{tol}_{1}=10^{-6}$. We stop the iterates of the outer Algorithm 5.1, when a maximum number of outer iterations maxiter $_{2}=100$ is reached or the normalized outer residual, that is the $\ell_{2}$ norm of (3.21) using $\vartheta^{n}$ and recomputing $\hat{x}$ is below a tolerance of $\mathrm{tol}_{2}=10^{-3}$.

\subsubsection{Direct $\ell_{1}$ learning}

Next we discuss the reduced Newton learning algorithm for $\ell_{1}$ problems as presented in Algorithm 4.2. In step (ii) of the algorithm, we need to perform the primal feasibility step which amounts to computing the minimizer of the lower level problem. For this, we use a a standard primal Newton algorithm with Armijo-type backtracking linesearch which takes on average 10-20 iterations to bring the normalized residual of the primal equation below a threshold of $\mathrm{tol}_{1}=10^{-9}$ ). In step (iii) of the algorithm we need to compute the dual feasibility step which we solve by using the Matlab mldivide command. We again use a positive definite approximation of the matrix $P$ in (4.35) in case it has negative eigenvalues, by flipping the signs of the negative eigenvalues. Furthermore we perform full steps on $\mu$ and $\vartheta_{i}$, $\forall i \in \mathcal{A}^{n}$ and an Armijo-type backracking linesearch on $\vartheta_{i}, \forall i \in \mathcal{I}^{n}$ using the higher level problem $\mathcal{E}(\vartheta)$ as the merit function. We set $\varepsilon=10^{-3}$ in the 4 -th order polynomial approximation of the $|\cdot|$ function in (4.5). We stop the algorithm when a maximum number of iterations maxiter $=100$ is reached or the normalized residual, i.e. the $\ell_{2}$ norm of the right hand side of the Newton equation in step (iv) devided by its number of elements is less a tolerance of $\mathrm{tol}_{2}=10^{-3}$.

\subsubsection{Results}

Table 1 shows the result of learning the optimal regularization parameters on natural images for various linear operators and noise levels using the iteratively reweighted $\ell_{2}$ learning algorithm $\left(\right.$ IRL- $\left.\ell_{2}\right)$ and the reduced Newton $\ell_{1}$ learning algorithm $\left(\mathrm{RNL}-\ell_{1}\right)$.

In general, one can see that the energy of the higher level problem $\mathcal{E}(\vartheta)$ decreases with the diversity of the filter banks and equivalently, the quality of the $\ell_{1}$ models increase with the diversity of the differentiation order included in the filter banks. Observe that the largest performance increase comes 


\begin{tabular}{|c|c|c|c|c|c|c|}
\hline Algorithm & IRL- & & & & & \\
\hline & & $=15$ & & $=25$ & & $=50$ \\
\hline Filters & $\mathrm{k}$ & $\mathcal{E}(\vartheta)$ & $\mathrm{k}$ & $\mathcal{E}(\vartheta)$ & $\mathrm{k}$ & $\mathcal{E}(\vartheta)$ \\
\hline $1^{\text {st }}$ & 107 & 163.19 & 145 & 303.21 & 191 & $\overline{602.83}$ \\
\hline $1^{s t}+2^{n d}$ & 119 & 152.98 & 190 & 282.91 & 174 & 563.86 \\
\hline$D C T 3$ & 132 & 148.79 & 141 & 272.60 & 183 & 545.46 \\
\hline$D C T 5$ & 101 & 147.67 & 150 & 268.12 & 506 & 529.83 \\
\hline Algorithm & RNL & & & & & \\
\hline & & 15 & & $=25$ & & $=50$ \\
\hline Filters & $\mathrm{k}$ & $\mathcal{E}(\vartheta)$ & $\mathrm{k}$ & $\mathcal{E}(\vartheta)$ & $\mathrm{k}$ & $\mathcal{E}(\vartheta)$ \\
\hline $1^{s t}$ & 8 & 162.87 & 24 & 302.69 & 16 & 601,88 \\
\hline $1^{s t}+2^{\text {nd }}$ & 18 & 152.45 & 33 & 282.02 & 43 & 562.44 \\
\hline$D C T 3$ & 12 & 147.55 & 20 & 270.62 & 37 & 542.90 \\
\hline$D C T 5$ & 16 & 144.69 & 44 & 265.41 & 100 & 525.97 \\
\hline
\end{tabular}

Table 1: Results for the $\ell_{1}$ learning algorithms on natural images. The table shows the number of Newton steps and the value of the higher- level problem $\mathcal{E}(\vartheta)$.

through adding second-order filters to the first-order derivative filters. We also performed experiments where we added first-order derivative filters to the DCT filter banks and it happened that the weights of the first-order filters where set to zero by the learning algorithm. This experiment suggests that the first-order filters and hence the classical total variation prior is not very suitable for natural images. In contrast, we observed that on randomly generated piecewise constant images the learning algorithm always preferred first-order filters over any additional higher order filter, which suggest that for piecewise constant images, the total variation is already a very good prior.

Comparing the results of the IRL- $\ell_{2}$ and RNL- $\ell_{1}$ algorithms, one can clearly see that RNL- $\ell_{1}$ needs far less Newton steps to converge. This can be explained by the fact that the IRL- $\ell_{2}$ algorithm performs a fixed-point iteration by solving a sequence of re-weighted $\ell_{2}$ learning problems and hence the overall algorithm is in principle a first-order algorithm. In contrast, the RNL- $\ell_{1}$ algorithm is a full Newton algorithm on the original $\ell_{1}$ learning problem and hence exhibits super-linear convergence. 


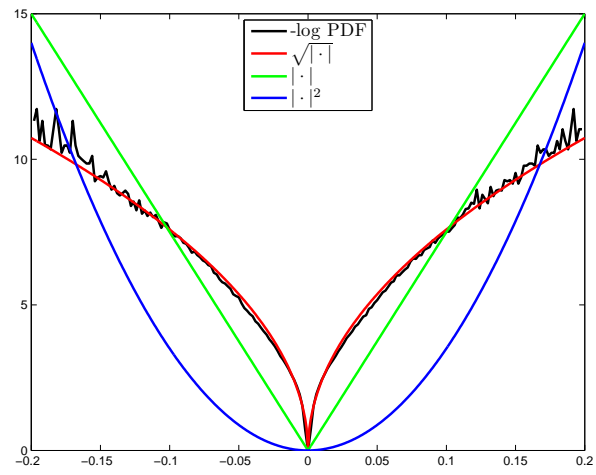

(a)

Figure 6: Negative log probability density function (PDF) of the filter response of a DCT5 filter applied to natural images. Note that the $\sqrt{|\cdot|}$ function provides the best fit to the heavy tailed shape of the true density function.

Furthermore, one can see that the RNL- $\ell_{1}$ algorithm stops at slightly smaller energies. This is explained by the fact that for a fixed value of $\varepsilon$ the function (4.5) utilized in the RNL- $\ell_{1}$ algorithm is a better approximation to the true $|\cdot|$ function than the Huber function (5.2) utilized in the IRL- $\ell_{2}$ algorithm. We also tried to us a smaller $\varepsilon$ in the IRL- $\ell_{2}$ which however lead to convergence problems.

\subsubsection{Learning of a non-convex $\ell_{\frac{1}{2}}$ model}

It is well known that the probability density function (PDF) of the responses of zero mean linear filters (e.g. DCT filters) on natural images have heavily tailed distributions [15]. Figure 6 plots the negative log PDF of last DCT5 filter shown in Figure 5 applied to natural images together with different model fits. One can clearly see that the $|\cdot|^{2}$ function provides a bad fit to the negative $\log \mathrm{PDF}$ which is consistent with the inferior performance of quadratic energies for image regularization. Although the $|\cdot|$ function provides a much better fit than the $|\cdot|^{2}$ function, the $\sqrt{|\cdot|}$ function represents an almost perfect model. However, while the $|\cdot|$ function is still convex the $\sqrt{|\cdot|}$ is non-convex which makes the lower problem much harder to solve.

Our aim is now to show that we can utilize the algorithms developed in this paper to learn the optimal regularization parameters of a model 
involving the non-convex $\ell_{\frac{1}{2}}$ quasi-norm. We shall see that this simple nonconvex model achieves excellent image denoising results very close to stateof-the-art algorithms.

The bilevel learning problem involving the non-convex $\ell_{\frac{1}{2}}$ quasi-norm is given by

$$
\left\{\begin{array}{l}
\min _{\vartheta \geq 0} \mathcal{E}(x(\vartheta))=\sum_{i=1}^{l}\left\|x_{i}(\vartheta)-g_{i}\right\|_{2}^{2} \\
\text { subject to } x_{i}(\vartheta)=\underset{x}{\operatorname{argmin}} 2 \sum_{k=1}^{q} \vartheta_{k}\left\|K_{k} x\right\|_{\frac{1}{2}}^{\frac{1}{2}}+\frac{1}{2}\left\|x-f_{i}\right\|_{2}^{2},
\end{array}\right.
$$

where $\left\|K_{k} x\right\|_{\frac{1}{2}}^{\frac{1}{2}}=\sum_{i=1}^{n} \sqrt{\left|\left(K_{k} x\right)_{i}\right|}$. In order to apply our learning algorithms, we need to regularize the above problem. Similar to (4.5) we use a locally regularized approximation of the $\sqrt{|\cdot|}$ function:

$$
n_{\varepsilon}(t)=\left\{\begin{array}{c}
-\frac{3 t^{4}}{32 \sqrt{\varepsilon^{7}}}+\frac{7 t^{2}}{16 \sqrt{\varepsilon^{3}}}+\frac{21 \sqrt{\varepsilon}}{32} \text { if }|t|<\varepsilon \\
\sqrt{|t|} \quad \text { else }
\end{array}\right.
$$

with derivatives

$$
\begin{gathered}
n_{\varepsilon}^{\prime}(t)=\left\{\begin{aligned}
-\frac{3 t^{3}}{8 \sqrt{\varepsilon^{7}}}+\frac{7 t}{8 \sqrt{\varepsilon^{3}}} & |t|<\varepsilon \\
\frac{t \sqrt{|t|^{3}}}{2} & \text { else, }
\end{aligned}\right. \\
n_{\varepsilon}^{\prime \prime}(t)=\left\{\begin{aligned}
-\frac{9 t^{2}}{8 \sqrt{\varepsilon^{7}}}+\frac{7}{8 \sqrt{\varepsilon^{3}}} & \text { if } \quad|t|<\varepsilon \\
-\frac{1}{4 \sqrt{|t|^{3}}} & \text { else, }
\end{aligned}\right. \\
n_{\varepsilon}^{\prime \prime \prime}(t)=\left\{\begin{array}{rll}
-\frac{9 t}{4 \sqrt{\varepsilon^{7}}} & \text { if } & |t|<\varepsilon \\
\frac{3 t}{8 \sqrt{|t|^{7}}} & \text { else. }
\end{array}\right.
\end{gathered}
$$

For learning, we use the reduced Newton Algorithm 4.2, which can be easily adapted to the $\ell_{\frac{1}{2}}$ setting by replacing the regularized $\ell_{1}$ norm with the regularized $\ell_{\frac{1}{2}}$ quasi-norm. We term the resulting algorithm the reduced Newton $\ell_{\frac{1}{2}}$ learning algorithm $\left(\mathrm{RNL}-\ell_{\frac{1}{2}}\right)$.

In our experiments we observe that the Hessian matrix in the $\ell_{\frac{1}{2}}$ case can have strongly negative eigenvalues and that computing a positive definite approximation of the Hessian by simply flipping the signs of the negative eigenvalues does not always lead to a very good second order approximation. This results in a worse convergence bahavior of the algorithm. We stop the 
algorithm after the normalized residual is below a tolerance of tol $=10^{-3}$, or a maximum number of iterations maxiter $=100$ is reached. The investigation of an improved Newton algorithm, for example the development of a trust region Newton method is subject to future work. For computing the primal feasibility step, we use the limited memory BFGS quasi-Newton method [20], where again for convergence reasons, we set $\varepsilon=10^{-2}$ in the $\varepsilon$ regularized $\sqrt{|\cdot|}$ function (5.3). The development of an algorithm that can handle smaller $\varepsilon$ is left to future work. Table 2 shows the results of apply-

\begin{tabular}{|c|c|c|c|c|c|c|}
\hline Algorithm & & $-\ell_{\frac{1}{2}}$ & & & & \\
\hline \multirow[b]{2}{*}{ Filters } & \multicolumn{2}{|c|}{$\sigma=15$} & \multicolumn{2}{|c|}{$\sigma=25$} & \multicolumn{2}{|c|}{$\sigma=50$} \\
\hline & $\mathrm{k}$ & $\mathcal{E}(\vartheta)$ & $\mathrm{k}$ & $\mathcal{E}(\vartheta)$ & $\mathrm{k}$ & $\overline{\mathcal{E}(\vartheta)}$ \\
\hline$D C T 3$ & 47 & 134.02 & 100 & 253.35 & 100 & 527.13 \\
\hline$D C T 5$ & 13 & 128.63 & 100 & 240.63 & 100 & 500.83 \\
\hline
\end{tabular}

Table 2: Results for the $\ell_{\frac{1}{2}}$ learning algorithm on natural images. of Newton steps and the value of the higher level problem $\mathcal{E}(\vartheta)$.

ing the RNL- $\ell_{\frac{1}{2}}$ to natural images using DCT3 and DCT5 filter banks and various noise levels. Observe that the RNL- $\ell_{\frac{1}{2}}$ algorithm takes significantly more iterations than the RNL- $\ell_{1}$ algorithm. However, as already said, our predominant aim is to show the potential of the non-convex $\ell_{\frac{1}{2}}$ model and hence also the limitations of the convex $\ell_{1}$ model. Comparing the function values of $\mathcal{E}(\vartheta)$ using the $\ell_{\frac{1}{2}}$ models to the function values when using $\ell_{1}$ models as shown in Table 1 we can see that the non-convex $\ell_{\frac{1}{2}}$ models lead to significantly lower function values which means that the $\ell_{\frac{1}{2}}$ can recover images which are closer to the ground-truth images.

\section{$5.2 \quad$ Testing}

In this section we use the learned models from the last section to evaluate their denoising performance on unseen images from the BSDS500 database [1]. Furthermore, we will show comparisons to related methods as well as stateof-the-art algorithms.

In this work, we inherently assumed that the noise level of the images is known in advance. We point out that this assumption is reasonable also for practical problems since in many cases, the noise level can be computed from the image acquisition process, can be specified by the user, or can be 
estimated by separate algorithms [19].

Having given the noise level, we compute the solution of the lower-level problems using the first-order primal-dual algorithm [4] with the preconditioning described in [25] in case of the $\ell_{1}$ models and used the limited memory BFGS quasi-Newton method [20] in case of the $\ell_{\frac{1}{2}}$ models. Note that for testing we require only a moderate accuracy of the minimizers of the lower-level problems and hence we stopped the algorithms after the change of the function value was below a threshold of tol $=10^{-3}$.

\subsubsection{Results of the $\ell_{1}$ model}

Figures 7, 8 and 9 show the denoising results of the learned $\ell_{1}$ models on natural images containing zero-mean Gaussian noise of various standard deviations, $\sigma \in\{15,25,50\}$. One can observe that larger filter banks consistently lead to a better image denoising performance, where in particular, the DCT filters are much better in recovering textured areas. Furthermore one can see that while the first-order filters lead to cartoon-like images (see the detail views in the last rows of the figures), the higher order filters lead to much more naturally appearing results.

From the experiments we can observe an interesting limitation of the $\ell_{1}$ models. While the step from simple first-order priors (i.e. the total variation) to higher order priors (e.g. second-order derivatives or DCT3) gives the largest performance increase, the performance seems to saturate when further increasing the diversity of the filter banks (e.g. from DCT3 to DCT5) and hence we expect that the performance of $\ell_{1}$ models cannot be improved much more by keep adding priors to the model. We do not think that this is due to a wrong set of priors (we also experimented with dictionary priors such as SVD and ICA priors) but is an inherent limitation of the $\ell_{1}$ model. Indeed, we will see that switching from the convex $\ell_{1}$ model to the non-convex $\ell_{\frac{1}{2}}$ model will overcome this limitation.

\subsubsection{Comparison between the $\ell_{1}$ model and the $\ell_{\frac{1}{2}}$ model}

Figure 10 shows a comparison between the convex $\ell_{1}$ model and the nonconvex $\ell_{\frac{1}{2}}$ model using the DCT5 filter bank for different noise levels. One can clearly see that the non-convex $\ell_{\frac{1}{2}}$ model leads to significantly better denoising results and the difference is higher for smaller noise levels. We can characterize the qualitative differences between the $\ell_{1}$ model and the $\ell_{\frac{1}{2}}$ model as follows: 
(i) The $\ell_{\frac{1}{2}}$ model leads to a better preservation of the contrast in the reconstructed image than the $\ell_{1}$ model. Let us interpret both models in terms of a shrinking process. It is known that the $\ell_{1}$ model performs in principle a soft-shrinkage of the coefficient which shrinks the coefficients independently of their strength. The $\ell_{\frac{1}{2}}$, however, performs a stronger shrinkage of smaller coefficients and a weaker shrinkage of larger coefficients which results in a better preservation of the contrast.

(ii) The $\ell_{1}$ model is not very successful in recovering homogeneous areas although it preserves textured regions very well. This effect comes from the convexity of the $\ell_{1}$ norm which cannot distinguish very well between homogeneous regions and textured regions. In contrast, the concave shape of the $\ell_{\frac{1}{2}}$ norm is much more successful in distinguishing textured and non-textured areas and hence gives better results.

(iii) As already pointed out above, further increasing the diversity of the $\ell_{1}$ model does not improve the denoising performance. In contrast, the performance of the $\ell_{\frac{1}{2}}$ can be further improved by increasing the diversity of the filter bank (see also Table 2).

In [27], Samuel and Tappen proposed a bilevel learning algorithm to learn the optimal filters (comparable to a dictionary) of the so-called Fields of Experts (FoE) model of Roth and Black [26]. The FoE model uses a sum of priors involving non-convex potential functions related to the negative log density of a Student-t distribution. The optimization algorithm is a plain gradient descend algorithm, where the gradients are computed using implicit differentiation. Since the FoE model has much more degrees of freedom as our simple models, one would expect that the FoE model would lead to better results. However, it turns out that our simple convex $\ell_{1}$ model leads to comparable and our non-convex $\ell_{\frac{1}{2}}$ model leads to significantly better results (see Figure 11 for an example). We do not exactly know the reason for the improved performance of our simpler model, but possibly, our Newton algorithms are distinctly more accurate in approximating (locally) optimal solutions than the gradient descend methods that are used in [27]. This fact justifies the use of Newton algorithms for this kind of learning problems.

\subsubsection{Comparison to state-of-the-art methods}

In our last experiment, we compare the results of our $\ell_{1}$ and $\ell_{\frac{1}{2}}$ models to state-of-the art algorithms. Figure 12 shows a comparison of the proposed 
models to the Fields of Experts (FoE) model of Roth and Black [26], the KSVD dictionary learning algorithm of Elad and Aharon [11], the recently proposed Gaussian mixture model (GMM) of Zoran and Weiss [33] and the BM3D algorithm of Dabov et al. [7] which define the current state-ofthe-art in image denoising. One can see that while the convex $\ell_{1}$ model cannot compete with the current state-of-the-art, the non-convex $\ell_{\frac{1}{2}}$ model is clearly state-of-the-art. Note that the two methods GMM and BM3D which are superior to our $\ell_{\frac{1}{2}}$ are much more involved. For example, the GMM method uses a generic image prior consisting of a Gaussian mixture model with 200 components, each of them specified by a $64 \times 64$ covariance matrix. Decomposing these covariance matrices into its eigenvectors, we end up with a total of 12800 filters whereas our $\ell_{\frac{1}{2}}$ model uses only 24 DCT5 filters. The BM3D method is still the best method on this example, although it can also lead to strange artifacts, as can be seen from the overemphasis of the stripe-like texture in the detail view in Figure 12.

\section{Conclusion and Outlook}

In this paper we have proposed semi-smooth Newton methods for learning the optimal regularization parameters in variational image denoising models including the smooth $\ell_{2}$ norm as well as the non-smooth $\ell_{1}$ norm. The parameters are learned in a way such that the minimizers of the variational models give the best approximation to given ground truth solutions. This naturally leads to a bilevel optimization approach with the higher level problem being a loss function that minimizes the error between the the solution of the lower level optimization problem (the variational model) and given ground truth data. We have analyzed the structure of the arising bilevel optimization problems and in case of a $\ell_{2}$ model with a single prior we were able to show that the problem is quasiconvex in the regularization parameter.

We have proposed and analyzed semi-smooth Newton methods that lead to efficient learning algorithms with guaranteed locally superlinear convergence. We tested the algorithms on natural image denoising problems using different noise levels and different sets of regularization priors. We have demonstrated the our proposed Newton algorithms can efficiently find optimal regularization parameters requiring approximately 20 Newton iterations on average.

Furthermore we have presented preliminary results on applying the bilevel learning framework to variational models including the non-smooth and non- 


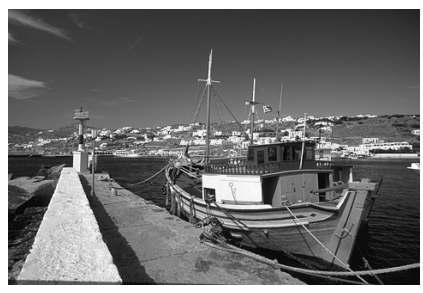

(a) clean

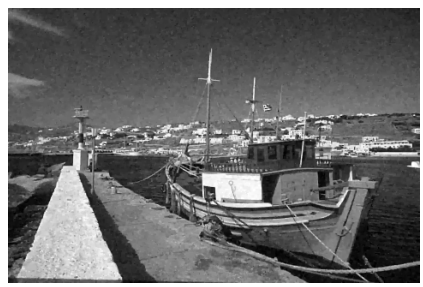

(d) $1^{s t}+2^{n d}(29.31)$

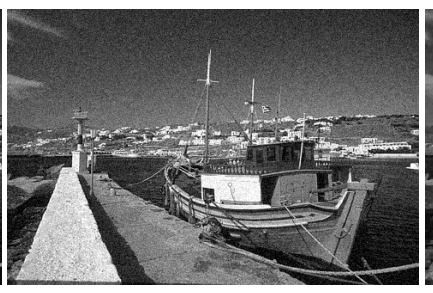

(b) noisy (24.61)

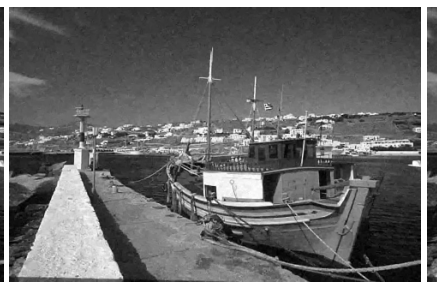

(e) $D C T 3(29.50)$

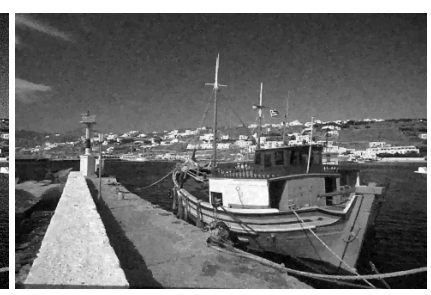

(c) $1^{\text {st }}(29.12)$

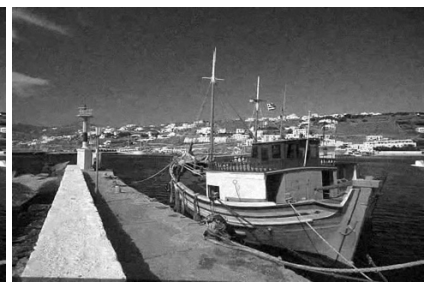

(f) $D C T 5(29.49)$

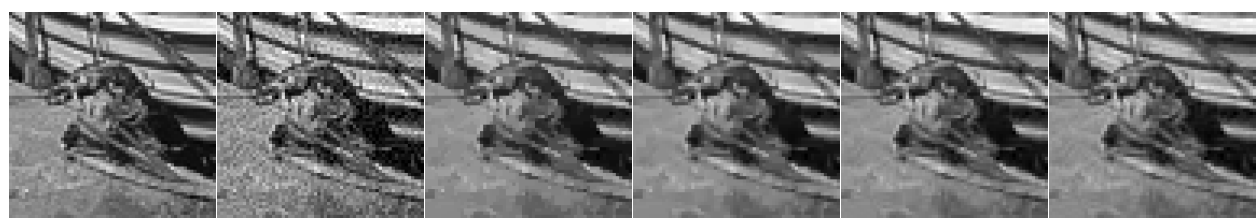
(g) clean
(h) noisy
(i) $1^{\text {st }}$
(j) $1^{\text {st }}+2^{\text {nd }}$
(k) $D C T 3$
(1) $D C T 5$

Figure 7: Image denoising performance of the trained $\ell_{1}$ model for a natural image and $\sigma=15$. The numbers shown in the brackets refer to PSNR values with respect to the clean image.

convex $\ell_{\frac{1}{2}}$ norm. In particular, we have shown that switching from the $\ell_{1}$ norm to the $\ell_{\frac{1}{2}}$ consistently improved the denoising performance over the $\ell_{1}$ models.

Future work should include the investigation of data fidelity terms different of quadratic ones and a further analysis of models incorporating the non-convex $\ell_{\frac{1}{2}}$ norm.

\section{A Proof of Theorem 4.3}

The proof is given in several steps.

(i) First we need to adress convergence of the solutions $\vartheta^{\varepsilon}$ to (4.4) as $\varepsilon \rightarrow$ 


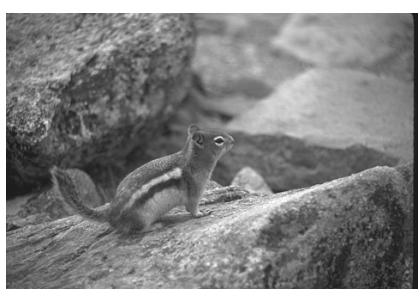

(a) clean

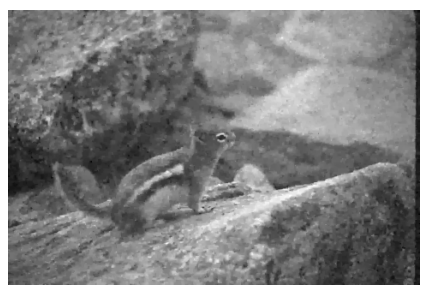

(d) $1^{\text {st }}+2^{\text {nd }}(28.73)$

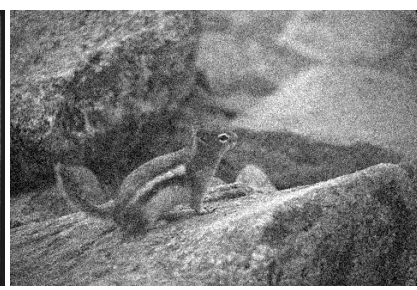

(b) noisy (20.17)

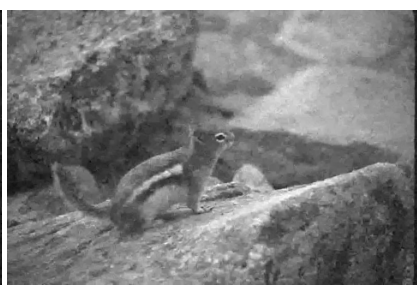

(e) $\operatorname{DCT} 3(28.81)$

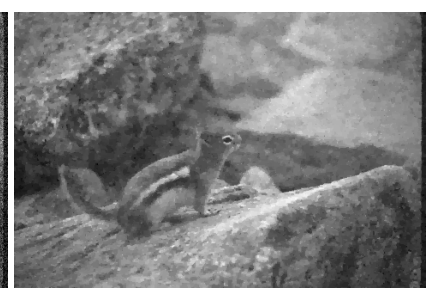

(c) $1^{\text {st }}(28.17)$

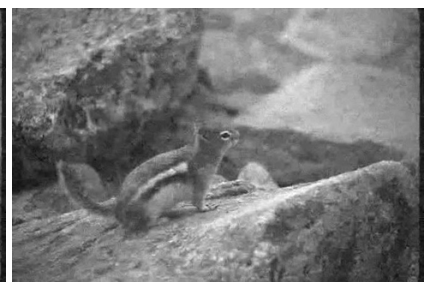

(f) $\operatorname{DCT} 5(28.93)$

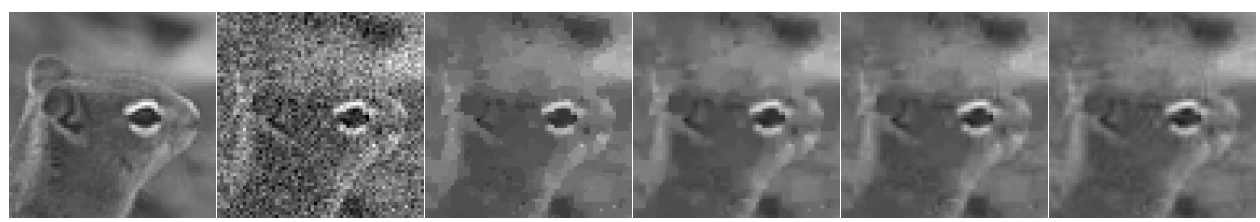

(g) clean

(h) noisy

(i) $1^{\text {st }}$

(j) $1^{s t}+2^{\text {nd }}$

(k) $D C T 3$

(1) $D C T 5$

Figure 8: Image denoising performance of the trained $\ell_{1}$ model for a natural image and $\sigma=25$. The numbers shown in the brackets refer to PSNR values with respect to the clean image.

$0^{+}$. It is not difficult to see that convergent subsequences of $\vartheta^{\varepsilon}$ converge to a solution of (4.1) but since the solutions to (4.1) are not unique, this may not be the desired one. For this reason we adapt Barbu's trick and introduce (only for the purpose of deriving the optimality condition) the auxiliary problem
(A.1) \{$$
\min _{\vartheta \geq 0}\|x(\vartheta)-g\|_{2}^{2}+\left\|\vartheta-\vartheta^{*}\right\|_{2}^{2}
$$$$
\text { subject to } x(\vartheta)=\arg \min _{x} \sum_{k=1}^{q} \vartheta_{k}\left\|K_{k} x\right\|_{1}+\frac{1}{2}\|x-f\|_{2}^{2} \text {, }
$$ 


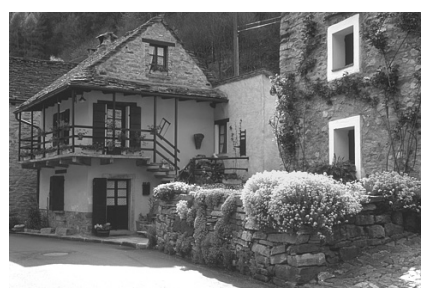

(a) clean

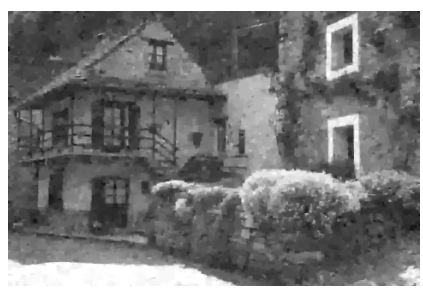

(d) $1^{\text {st }}+2^{n d}(23.15)$

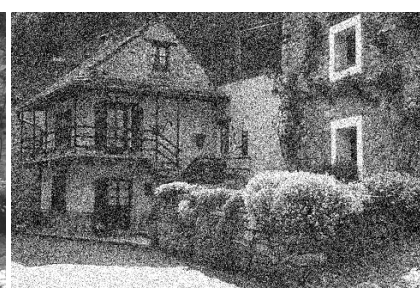

(b) noisy (14.15)

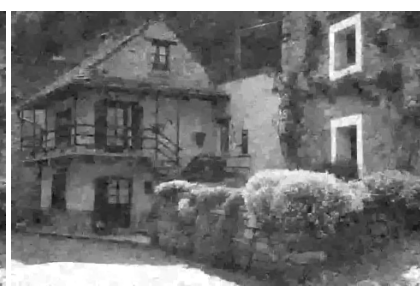

(e) $D C T 3(23.28)$

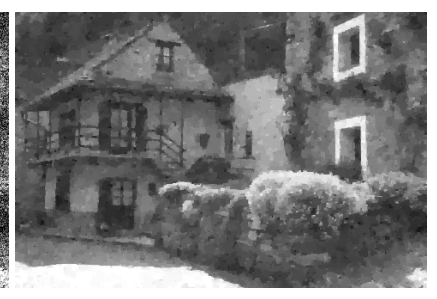

(c) $1^{\text {st }}(22.97)$

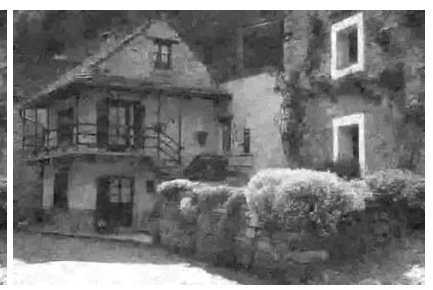

(f) $D C T 5(23.31)$

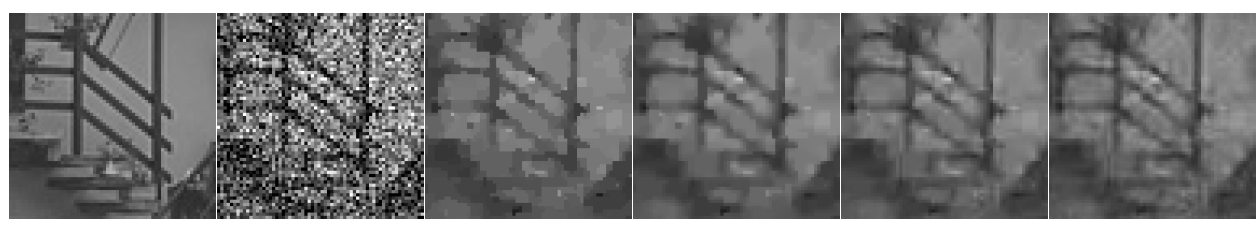
(g) clean
(h) noisy
(i) $1^{s t}$
(j) $1^{s t}+2^{n d}$
(k) $D C T 3$
(1) $D C T 5$

Figure 9: Image denoising performance of the trained $\ell_{1}$ model for a natural image and $\sigma=50$. The numbers shown in the brackets refer to PSNR values with respect to the clean image.

and the auxiliary regularized problem

(A.2)

$$
\left\{\begin{array}{l}
\min _{\vartheta \geq 0}\|x(\vartheta)-g\|_{2}^{2}+\left\|\vartheta-\vartheta^{*}\right\|_{2}^{2} \\
\text { subject to } x(\vartheta)=\arg \min _{x} \sum_{k=1}^{q} \vartheta_{k} \sum_{i=1}^{m} n_{\varepsilon}\left(\left(K_{k} x\right)_{i}\right)+\frac{1}{2}\|x-f\|_{2}^{2}
\end{array}\right.
$$

Adding the term $\left\|\vartheta-\vartheta^{*}\right\|_{2}^{2}$ to the cost has no effect on the discussion preceeding the statement of the theorem. Problem (A.1) has $\vartheta^{*}$ as unique solution. The necessarily optimality condition for (A.2) con- 


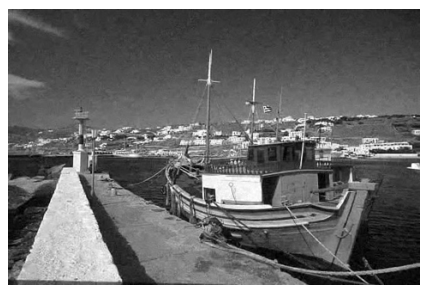

(a) $\ell_{1}(29.49)$

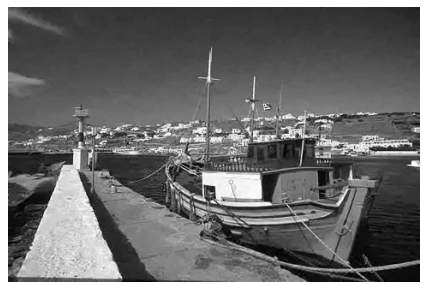

(d) $\ell_{\frac{1}{2}}(30.24)$

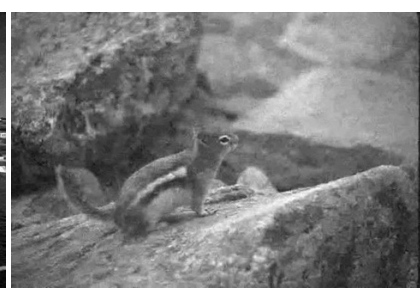

(b) $\ell_{1}(28.93)$

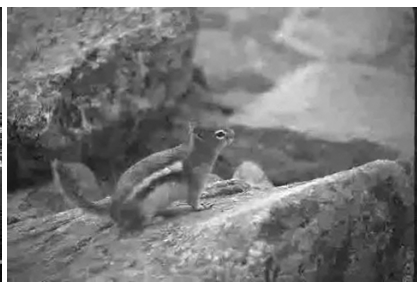

(e) $\ell_{\frac{1}{2}}(29.19)$

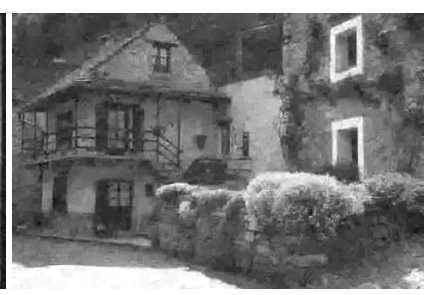

(c) $\ell_{1}(23.31)$

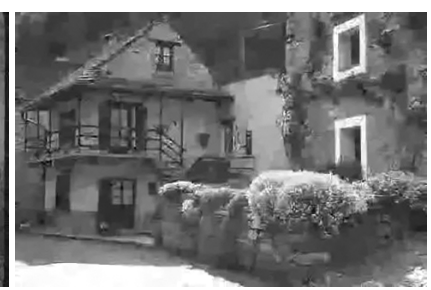

(f) $\ell_{\frac{1}{2}}(23.42)$

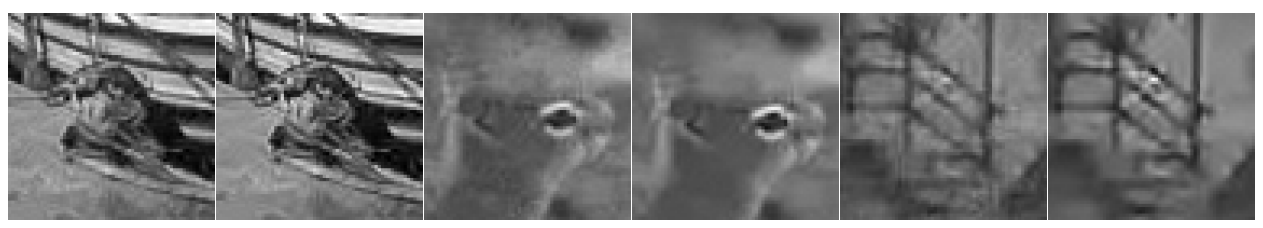
(g) $\ell_{1}$
(h) $\ell_{\frac{1}{2}}$
(i) $\ell_{1}$
(j) $\ell_{\frac{1}{2}}$
(k) $\ell_{1}$
(l) $\ell_{\frac{1}{2}}$

Figure 10: Comparison between the convex $\ell_{1}$ model and the non-convex $\ell_{\frac{1}{2}}$ model for different noise levels and using DCT5 filters. The numbers shown in the brackets refer to PSNR values with respect to the clean image.

sists of the first two equations in (4.13) and

(A.3)

$\left(\left\langle N_{\varepsilon}^{\prime}\left(K_{k} x_{\varepsilon}\right), K_{k} p_{\varepsilon}\right\rangle+2\left(\vartheta_{\varepsilon, k}-\vartheta_{k}^{*}\right)\right)\left(\vartheta_{k}-\vartheta_{\varepsilon, k}\right) \geq 0$, for all $\vartheta_{k} \geq 0, k=1, \ldots, q$.

Let $\left\{\vartheta_{\varepsilon}\right\}_{\varepsilon>0}$ denote a family of solutions to (A.2). Since $\vartheta^{*}$ is suboptimal for (A.2) we obtain that

$$
\left\|\vartheta_{\varepsilon}-\vartheta^{*}\right\|_{2} \leq\left\|x\left(\vartheta^{*}\right)-g\right\|_{2}+\left\|\vartheta^{*}\right\|_{2}
$$

and therefore $\left\{\vartheta_{\varepsilon}\right\}_{\varepsilon>0}$ is bounded. By the first equation in (4.13) the family $x_{\varepsilon}=x\left(\vartheta_{\varepsilon}\right)$ is bounded as well. Hence there exists a subsequence, denoted by the same symbol, and $\bar{\vartheta} \in \mathbb{R}^{q}$ such that 


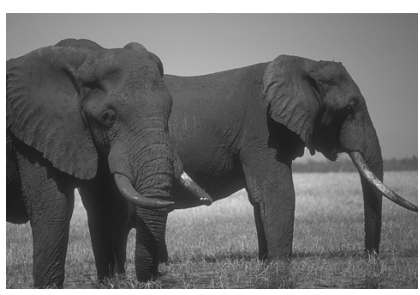

(a) clean

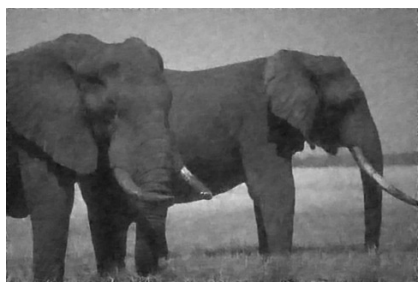

(c) FoE-bi (29.33)

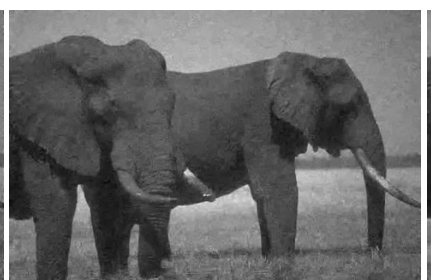

(d) $\ell_{1}(29.33)$

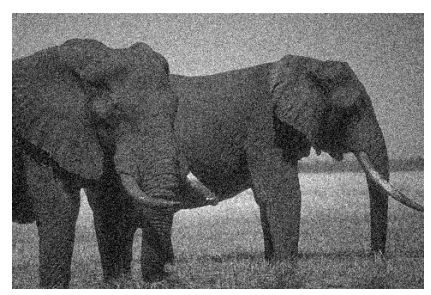

(b) $\operatorname{noisy}(20.17)$

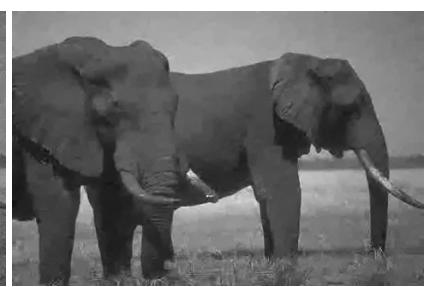

(e) $\ell_{\frac{1}{2}}(29.70)$

Figure 11: Comparison between our $\ell_{1}$ and $\ell_{\frac{1}{2}}$ models using DCT5 filters and the bilelvel-optimized Fields of Experts (FoE-bi) model [27]. The numbers shown in the brackets refer to PSNR values with respect to the clean image.

$\lim _{\varepsilon \rightarrow 0^{+}} \vartheta_{\varepsilon}=\bar{\vartheta}$ and $\lim _{\varepsilon \rightarrow 0^{+}} x_{\varepsilon}\left(\vartheta_{\varepsilon}\right)=x(\bar{\vartheta})$. Taking the limit $\varepsilon \rightarrow 0^{+}$ in

$$
\left\|x_{\varepsilon}\left(\vartheta_{\varepsilon}\right)-g\right\|_{2}^{2}+\left\|\vartheta_{\varepsilon}-\vartheta^{*}\right\|_{2}^{2} \leq\left\|x_{\varepsilon}(\vartheta)-g\right\|_{2}^{2}+\left\|\vartheta-\vartheta^{*}\right\|_{2}^{2}
$$

where $x_{\varepsilon}(\vartheta)=\arg \min _{x} \sum_{k=1}^{q} \vartheta_{k} \sum_{i=1}^{m} n_{\varepsilon}\left(\left(K_{k} x\right)_{i}\right)+\frac{1}{2}\|x-f\|_{2}^{2}$,

we obtain

$$
\|x(\bar{\vartheta})-g\|_{2}^{2}+\left\|\bar{\vartheta}-\vartheta^{*}\right\|_{2}^{2} \leq\|x(\vartheta)-g\|_{2}+\left\|\vartheta-\vartheta^{*}\right\|_{2}^{2}
$$

for all $\vartheta \geq 0$, where $x(\vartheta)=\arg \min _{x} \sum_{k=1}^{q} \vartheta_{k}\left\|K_{k} x\right\|_{1}+\frac{1}{2}\|x-f\|_{2}^{2}$. By construction this implies that $\vartheta=\vartheta^{*}$.

Let us henceforth set $\left(\lambda_{\varepsilon}\right)_{k}=N_{\varepsilon}^{\prime}\left(K_{k} x_{\varepsilon}\right)$, and $\xi_{\varepsilon}=\sum_{k=1}^{q} \vartheta_{\varepsilon, k} K_{k}^{T} N_{\varepsilon}^{\prime \prime}\left(K_{k} x_{\varepsilon}\right) K_{k} p_{\varepsilon}$.

(ii) By (4.13) and the properties of $n_{\varepsilon}$ the families $\left\{p_{\varepsilon}\right\}_{\varepsilon>0},\left\{\left(\lambda_{\varepsilon}\right)_{k}\right\}_{\varepsilon>0}$, and $\left\{\xi_{\varepsilon}\right\}_{\varepsilon>0}$ are bounded. Note that the boundedness of $\left\{\xi_{\varepsilon}\right\}_{\varepsilon>0}$ follows 


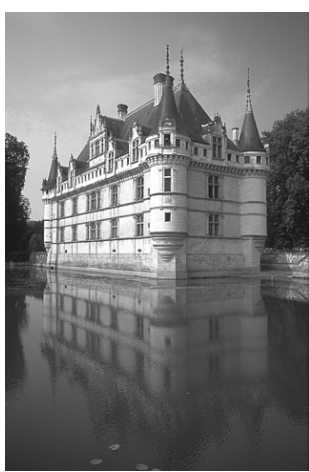

(a) clean

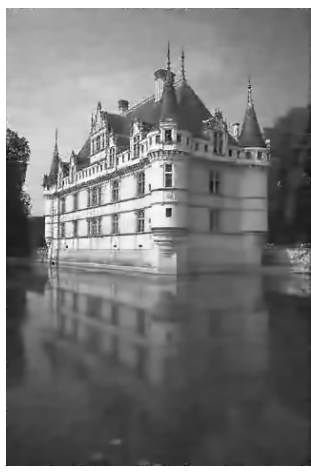

(e) $\operatorname{FoE}(28.72)$

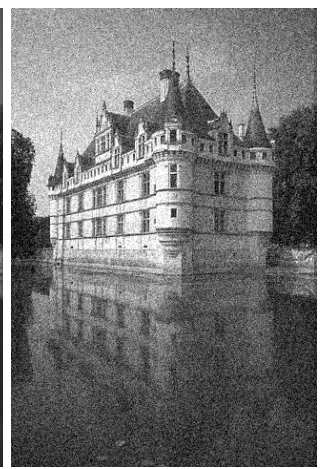

(b) noisy (20.17)

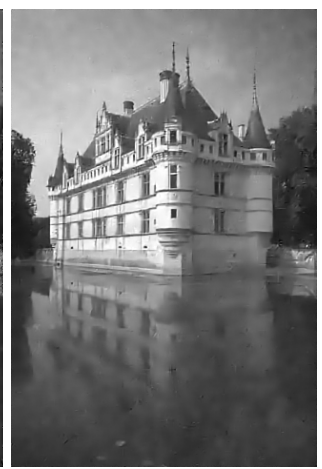

(f) $\operatorname{KSVD}(29.19)$

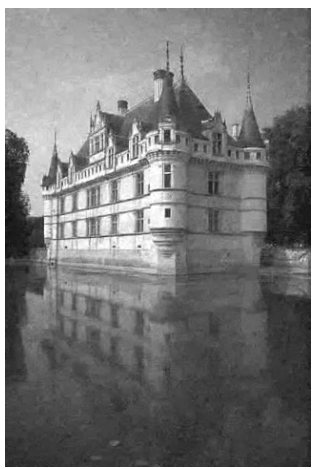

(c) $\ell_{1}(28.46)$

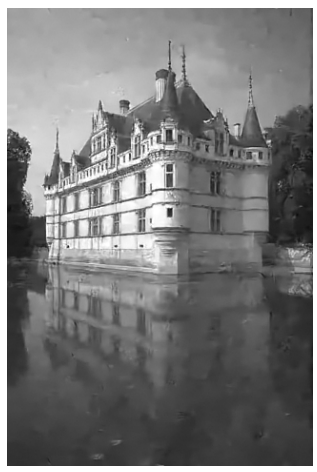

(g) $\operatorname{GMM}(29.48)$

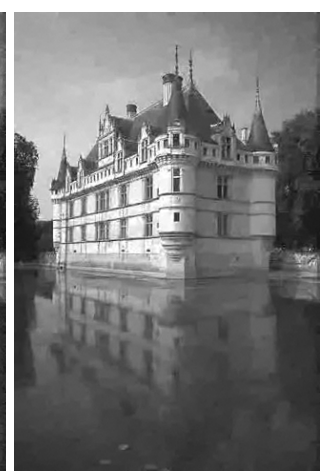

(d) $\ell_{\frac{1}{2}}(29.26)$

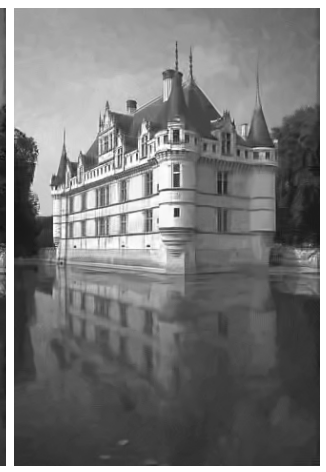

(h) $\operatorname{BM} 3 \mathrm{D}(29.53)$

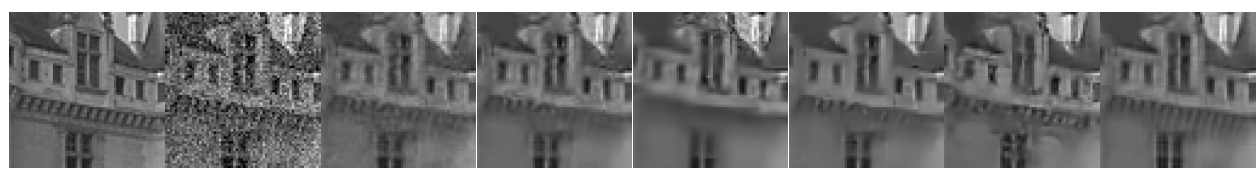
(i) clean
(j) noisy
(k) $\ell_{1}$
(l) $\ell_{\frac{1}{2}}$
(m) FoE
(n) KSVD
(o) GMM (p) BM3D

Figure 12: Comparison between the proposed $\ell_{1}$ and $\ell_{\frac{1}{2}}$ models to the Fields of Experts (FoE) model of Roth and Black [26], the KSVD dictionary learning algorithm of Elad and Aharon [11], the recently proposed Gaussian mixture model (GMM) of Zoran and Weiss [33] and the BM3D algorithm of Dabov et al. [7]. The numbers shown in the brackets refer to PSNR values with respect to the clean image.

from the adjoint equation, since $\left\{p_{\varepsilon}\right\}_{\varepsilon>0}$ is bounded. Hence, possibly 
after taking another subsequence, there exist $p, \lambda_{k}, k=1, \ldots, q$, and $\xi$ such that

$$
\left(p_{\varepsilon}, \lambda_{\varepsilon, k}, \xi_{\varepsilon}\right) \longrightarrow(p, \lambda, \xi) \text { as } \varepsilon \rightarrow 0^{+} .
$$

We can now pass to the limit in the first and second equation of (4.13) and in (A.3) to obtain the first, third and fourth equation of (4.14). Moreover $0 \leq \lim _{\varepsilon \rightarrow 0^{+}}\left\langle\xi_{\varepsilon}, p_{\varepsilon}\right\rangle=\langle\xi, p\rangle$, which gives the fifth assertion in (4.14). Passing to the limit in $\lambda_{\varepsilon}$ we find the second assertion of (4.14). Taking the inner product of the adjoint equation with $p_{\varepsilon}$ and passing to the limit we obtain the sixth equation in (4.14).

(iii) To verify the last two claims, we note at first that by the adjoint equation

$$
\left\|p_{\varepsilon}\right\|_{2}^{2}+\left|\sum_{k=1}^{q} \vartheta_{\varepsilon, k}\left\langle N_{\varepsilon}^{\prime \prime}\left(K_{k} x_{\varepsilon}\right) K_{k} p_{\varepsilon}, K_{k} p_{\varepsilon}\right\rangle\right| \leq\left\|x_{\varepsilon}-g\right\|\left\|p_{\varepsilon}\right\|_{2} .
$$

Consequently $\left\{\sum_{k=1}^{q} \vartheta_{\varepsilon, k}\left\|\sqrt{N_{\varepsilon}^{\prime \prime}\left(K_{k} x_{\varepsilon}\right)} K_{k} p_{\varepsilon}\right\|_{2}^{2}\right\}_{\varepsilon>0}$ is bounded. We have

$$
\begin{aligned}
\left|\left\langle\xi_{\varepsilon}, x_{\varepsilon}\right\rangle\right| & =\left|\sum_{k=1}^{q} \vartheta_{\varepsilon, k}\left\langle N_{\varepsilon}^{\prime \prime}\left(K_{k} x_{\varepsilon}\right) K_{k} p_{\varepsilon}, K_{k} x_{\varepsilon}\right\rangle\right| \\
& \leq \sum_{i=1}^{q} \vartheta_{\varepsilon, k}\left\|\sqrt{N_{\varepsilon}^{\prime \prime}\left(K_{k} x_{\varepsilon}\right)} K_{k} p_{\varepsilon}\right\|_{2}\left\|\sqrt{N_{\varepsilon}^{\prime \prime}\left(K_{k} x_{\varepsilon}\right)} K_{k} x_{\varepsilon}\right\|_{2} \\
& \leq\left(\sum_{k=1}^{q} \vartheta_{\varepsilon, k}\left\|\sqrt{N_{\varepsilon}^{\prime \prime}\left(K_{k} x_{\varepsilon}\right)} K_{k} p_{\varepsilon}\right\|_{2}^{2}\right)^{\frac{1}{2}}\left(\sum_{k=1}^{q} \vartheta_{\varepsilon, k}\left\|\sqrt{N_{\varepsilon}^{\prime \prime}\left(K_{k} x_{\varepsilon}\right)} K_{k} x_{\varepsilon}\right\|_{2}^{2}\right)^{\frac{1}{2}} \stackrel{\varepsilon \rightarrow 0^{+}}{\rightarrow} 0,
\end{aligned}
$$

by the properties of $n_{\varepsilon}^{\prime \prime}$. Therefore $\left|\left\langle\xi, x^{*}\right\rangle\right|=\lim _{\varepsilon \rightarrow 0^{+}}\left|\left\langle\xi_{\varepsilon}, x_{\varepsilon}\right\rangle\right|=0$ which is the seventh claim in (4.14). To verify the last one we set $\mathcal{I}_{\varepsilon, k}=\left\{i:\left|\left(K_{k} x_{\varepsilon}\right)_{i}\right|<\varepsilon\right\}$ and find

$$
\begin{aligned}
0 & \leq \sum_{i=1}^{m}\left|\left(K_{k} p_{\varepsilon}\right)_{i}\right|\left(1-\left|\left(\lambda_{\varepsilon, k}\right)_{i}\right|\right) \\
& =\sum_{i \in \mathcal{I}_{\varepsilon, k}}^{m}\left|\left(K_{k} p_{\varepsilon}\right)_{i}\right|\left(1-\left|\frac{3}{2 \varepsilon}\left(K_{k} x\right)_{i}-\frac{1}{2 \varepsilon^{3}}\left(K_{k} x\right)_{i}^{3}\right|\right) \\
& \leq \sum_{i \in \mathcal{I}_{\varepsilon, k}}^{m}\left|\sqrt{n^{\prime \prime}\left(K_{k} x_{\varepsilon}\right)}\left(K_{k} p_{\varepsilon}\right)_{i}\right| \frac{1}{\sqrt{n^{\prime \prime}\left(\left(K_{k} x_{\varepsilon}\right)_{i}\right)}}\left(1-\left|\frac{3}{2 \varepsilon}\left(K_{k} x_{\varepsilon}\right)_{i}-\frac{1}{2 \varepsilon^{3}}\left(K_{k} x_{\varepsilon}\right)_{i}^{3}\right|\right) \\
& \leq\left\|\sqrt{n^{\prime \prime}\left(K_{k} x_{\varepsilon}\right)}\left(K_{k} p_{\varepsilon}\right)\right\|_{2}\left(\sum_{i \in \mathcal{I}_{\varepsilon, k}} \frac{1}{n^{\prime \prime}\left(\left(K_{k} x_{\varepsilon}\right)_{i}\right)}\left(1-\left|\frac{3}{2 \varepsilon}\left(K_{k} x_{\varepsilon}\right)_{i}-\frac{1}{2 \varepsilon^{3}}\left(K_{k} x_{\varepsilon}\right)_{i}^{3}\right|\right)^{2}\right)^{\frac{1}{2}}
\end{aligned}
$$


Utilizing that $n^{\prime \prime}(t)=-\frac{3}{2 \varepsilon^{3}} t^{2}+\frac{3}{2 \varepsilon}$, for $|t|<\varepsilon$ one argues that $\lim _{\varepsilon \rightarrow 0^{+}} \sup _{|t| \leq \varepsilon} \frac{1}{n^{\prime \prime}(t)}(1-$ $\left.\left|\frac{3}{2 \varepsilon} t-\frac{1}{2 \varepsilon^{3}} t^{3}\right|\right)^{2}=0$ and hence $\sum_{i=1}^{\mu}\left(K_{k} p\right)_{i}\left(1-\left|\left(\lambda_{i}\right)_{i}\right|\right)=0$, as desired.

\section{References}

[1] P. Arbelaez, M. Maire, C. Fowlkes, and J. Malik. Contour detection and hierarchical image segmentation. IEEE Trans. Pattern Anal. Mach. Intell., 33(5):898-916, May 2011.

[2] J. Bard. Practical Bilevel Optimization. Kluwer Academic Publishers, Dordrecht, 1998.

[3] A. Chambolle and P.-L. Lions. Image recovery via total variation minimization and related problems. Numer. Math., 76:167-188, 1997.

[4] A. Chambolle and T. Pock. A first-order primal-dual algorithm for convex problems with applications to imaging. Journal of Mathematical Imaging and Vision, 40(1):120-145, 2010.

[5] T. F. Chan, G. H. Golub, and P. Mulet. A nonlinear primal-dual method for total variation-based image restoration. SIAM J. Sci. Comput., 20(6):1964-1977, May 1999.

[6] R. Chaney. Second-order necessary conditions in constrained semismooth optimization. SIAM J. Journal on Control and Optimization, 25(4):1072-1081, 1987.

[7] K. Dabov, A. Foi, V. Katkovnik, and K. Egiazarian. Image restoration by sparse $3 \mathrm{~d}$ transform-domain collaborative filtering. Proceedings of SPIE Electronic Imaging, 2008.

[8] I. Daubechies, R. DeVore, M. Fornasier, and C. S. Güntürk. Iteratively reweighted least squares minimization for sparse recovery. Comm. Pure Appl. Math., 63(1):1-38, 2010.

[9] J. C. de los Reyes. Optimal control of a class of variational inequalities of the second kind. SIAM J. Control and Optimization, 49(4):1629$1658,2011$.

[10] A. Doicu, T. Trautmann, and T. Schreier. Numerical Regularization for Atmospheric Inverse Problems. Springer-Verlag, 2010. Grundlehren der Mathematischen Wissenschaften, Band 132. 
[11] M. Elad and M. Aharon. Image denoising via sparse and redundant representations over learned dictionaries. IEEE Transactions on Image Processing, 15(12):3736-3745, 2006.

[12] H. W. Engl, M. Hanke, and A. Neubauer. Regularization of Inverse Problems, volume 375 of Mathematics and its Applications. Kluwer Academic Publishers Group, Dordrecht, 1996.

[13] W. Hinterberger, M. Hintermüller, K. Kunisch, M. Von Oehsen, and O. Scherzer. Tube methods for bv regularization. J. Math. Imaging Vis., 19(3):219-235, November 2003.

[14] M. Hintermüller and G. Stadler. An infeasible primal-dual algorithm for total bounded variation-based inf-convolution-type image restoration. SIAM J. Sci. Comput., 28(1):1-23, January 2006.

[15] J. Huang and D. Mumford. Statistics of natural images and models. In IEEE Conference on Computer Vision and Pattern Recognition (CVPR1999), pages 541-547, Fort Collins, CO, USA, 1999.

[16] K. Ito and K. Kunisch. Newton's method for a class of weakly singular optimal control problems. SIAM Journal on Optimization, 10(3):896, 2000 .

[17] K. Ito and K. Kunisch. Lagrange Multiplier Approach to Variational Problems and Applications. SIAM, Philadelphia, 2008.

[18] T. Kato. Perturbation theory for linear operators. Springer-Verlag, Berlin, second edition, 1976. Grundlehren der Mathematischen Wissenschaften, Band 132.

[19] C. Liu, W. T. Freeman, R. Szeliski, and S. B. Kang. Noise estimation from a single image. IEEE Conference on Computer Vision and Pattern Recognition (CVPR2006), pages 901-908, 2006.

[20] D. C. Liu and J. Nocedal. On the limited memory BFGS method for large scale optimization. Mathematical Programming, 45(1):503-528, 1989.

[21] D.G. Luenberger and Y. Yinyu. Linear and Nonlinear Programming. Springer, third edition, 2008.

[22] Jorge Nocedal and Stephen J. Wright. Numerical Optimization. Springer, 2000. 
[23] J.V. Outrata. A generalized mathematical program with equilibrium constraints. SIAM J. Control Optim., 38:16231638, 2000.

[24] G. Peyré and J. Fadili. Learning analysis sparsity priors. In Proc. of Sampta'11, 2011.

[25] T. Pock and A Chambolle. Diagonal preconditioning for first order primal-dual algorithms. In International Conference of Computer Vision (ICCV 2011), 2011.

[26] S. Roth and M. J. Black. Fields of experts. International Journal of Computer Vision, 82(2):205-229, 2009.

[27] K. G. G. Samuel and M.F. Tappen. Learning optimized map estimates in continuously-valued mrf models. In IEEE Conference on Computer Vision and Pattern Recognition (CVPR2009), 2009.

[28] M. F. Tappen, C. Liu, E. H. Adelson, and W. T. Freeman. Learning gaussian conditional random fields for low-level vision. In IEEE Conference on Computer Vision and Pattern Recognition (CVPR2007), pages $1-8,2007$.

[29] Marshall F. Tappen. Utilizing variational optimization to learn markov random fields. In IEEE Conference on Computer Vision and Pattern Recognition (CVPR2007), pages 1-8, 2007.

[30] M. Ulbrich. Semismooth Newton Methods for Variational Inequalities and Constrained Optimal Control Problems in Function Spaces. MOSSIAM Series in Optimization. SIAM, Philadelphia, 2011.

[31] S. Vaiter, G. Peyré, C. Dossal, and J. Fadili. Robust sparse analysis regularization. CoRR, abs/1109.6222, 2011.

[32] C. Vogel and M. Oman. Iteration methods for total variation denoising. SIAM J. Sci. Comp., 17:227-238, 1996.

[33] D. Zoran and Y. Weiss. From learning models of natural image patches to whole image restoration. Computer Vision, IEEE International Conference on, 0:479-486, 2011. 